\title{
On Evolution Equations for Moving Domains
}

\author{
G. Prokert
}

\begin{abstract}
We introduce a general framework for the description of the autonomous motion of closed surfaces which are diffeomorphic images of spheres. The governing surface motion laws are in general nonlocal and lead therefore to nonlocal evolution equations for a perturbation function on a fixed reference domain. Although this evolution equation is fully nonlinear, the invariance of the problem with respect to a certain class of reparametrizations and the corresponding chain rule allow a quasilincarization of the evolution equation. Hence, as far as short-time existence and uniqueness of the solution and stability of equilibria are concerned, the analysis of the problems is reduced to the study of their linearizations and the application of known techniques for quasilinear Cauchy problems. Using a priori estimates and Galerkin approximations in Sobolev spaces, both parabolic and first-order hyperbolic equations can be treated. In the case of parabolic problems, the smoothing property of the evolution can be proved.

This general approach can be applied to a broad class of moving boundary problems. We will briefly discuss Hele-Shaw flow and Stokes flow driven by surface tension as well as classical Hele-Shaw flow with advancing liquid boundary as examples for parabolic evolutions.
\end{abstract}

Keywords: Free boundary problems, surface motion laws, nonlocal parabolic evolution equations

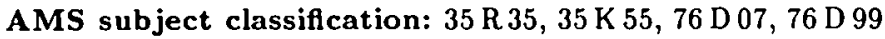

\section{Introduction}

A well-known and successful approach in the analytic treatment of free and moving boundary problems is the so-called direct mapping method (see, e.g., $[1,5,7,15,17$, $18,26])$. It is based on a correspondence between the unknown or moving domain and a real-valued continuous function on the boundary of a fixed reference domain. The original problem is reformulated as a problem of determining this unknown function. This method is particularly suitable for moving boundary problems which have the structure of a surface motion law, i.e. where the evolution of the moving boundary at a given time is completely determined by the domain at this time. In this case, the reformulated problem typically has the form of a nonlocal, nonlinear Cauchy problem which can be treated in an appropriate fixed function space by a variety of techniques depending on the particular situation. The properties of the solution to the Cauchy problem such as existence intervals, uniqueness, and smoothness, can be immediately translated into corresponding results for the original moving boundary problem.

G. Prokert: Universität Gesamthochschule Kassel, FB 17 Mathematik/Informatik, HeinrichPlett-Str. 40, D - 34109 Kassel; e-mail: prokert@mathematik.uni-kassel.de 
The main difficulty in this approach lies in the nonlinear and nonlocal character of the occurring evolution equation. In many cases, these equations are "fully nonlinear" in the sense that there is neither a semilinear nor an obvious quasilinear structure. However, in an abstract sense one can speak about parabolic or hyperbolic problems, depending on corresponding properties of the linearized problems, e.g. with respect to coercivity or energy estimates.

In the case of parabolic equations, the problems mentioned above can be overcome using the method of maximal regularity $[3,23]$. However, the general results on fully nonlinear equations provided by this theory are not completely satisfactory because they do not yield a proof of the smoothing effect of the evolution, i.e. the fact that the solution is a $C^{\infty}$-function in space for all positive times which is expected in a parabolic evolution. Moreover, the application of the theory of maximal regularity imposes strong restrictions on the choice of the function spaces which can lead to considerable technical complications.

Loosely speaking, the proof of the smoothing property for fully nonlinear parabolic initial value problems can be given by two approaches: One of them is embedded in the framework of the maximal regularity method and uses additional symmetry properties of the evolution equation (sce $[3,4,16])$. The other one goes back to Eidel'man [13] and is based on replacing the original evolution equation by a quasilinear parabolic system which is obtained by differentiating the original equation with respect to the space variable and using a chain rule which links spatial derivatives of the evolution operator to spatial derivatives of its argument. In the case of nonlocal evolutions, however, such a chain rule is itself an expression for certain invariances of the problem. This approach has been applied to parabolic moving boundary problems in $[18,26]$ and to a hyperbolic problem in [8]. Both methods for proving the smoothing effect share two disadvantages: As they rely on symmetries, they are applicable only to problems which have such properties (e.g. invariance with respect to rotations or translations), and there are unnatural geometric restrictions that have to be imposed on the moving domains (their boundaries have to be graphs over a suitable reference manifold).

It is the aim of this article to introduce a related general technique which avoids both disadvantages for domains that are diffeomorphic to balls. The unit ball is chosen as reference domain, and the evolution of the moving domain is represented, via a given smooth diffeomorphism, by the evolution of a perturbation of this ball. The basic idea is to consider the evolution of the perturbation as depending also on the diffeomorphism [19]. This leads to a non-uniqueness in the sense that the same domain can be described by a family of perturbations with corresponding diffeomorphisms. Clearly, the evolution is independent of the choice of a representant in this family. This invariance property implies a chain rule which can be applied for a quasilinearization in the way described above. It has to be pointed out that it is of a purely geometric nature and holds independently of invariances in the original moving boundary problem.

By this technique, the existence and uniqueness proof for short-time solutions of a broad class of moving boundary problems can essentially be reduced to the derivation of a priori estimates for the linearized problem in Sobolev spaces of sufficiently high order. Using these estimates, one can give the proof by standard arguments via Galerkin approximations. In the case of parabolic problems, the smoothing effect can be shown 
by a bootstrapping argument which is also based on the chain rule.

Finally, applications of the described approach to three well-known surface motion laws are discussed which lead to parabolic evolution equations, namely, the problems of Stokes flow and Hele-Shaw flow driven by surface tension and to classical Hele-Shaw flow with advancing liquid and a general interior driving mechanism. It is intended to describe applications that lead to hyperbolic evolution equations in a forthcoming paper.

\section{The evolution equation}

We consider domain evolution problems of a rather general type, which can informally be described in the following way: Given an initial domain $\Omega(0) \subset \mathbb{R}^{N}$ and an operator $\mathcal{U}$ which assigns to any (sufficiently smooth) domain $\Omega$ a vector field on its boundary:

$$
\mathcal{U}(\Omega): \partial \Omega \longrightarrow \mathbb{R}^{N}
$$

one looks for a parametrized family $\{\Omega(t)\}$ of domains $\Omega(t), t \in[0, T]$, such that the evolution is sufficiently smooth and the motion of the boundary hypersurface $\Gamma(t)=$ $\partial \Omega(t)$ is given by

$$
V_{n}(t)=\mathcal{U}(\Omega(t)) \cdot n(t) \quad \text { on } \Gamma(t),
$$

where $V_{n}(t)$ denotes the normal velocity of the boundary $\Gamma(t)$ at time $t$, and $n(t)$ is the outer unit normal field on $\Gamma(t)$. Condition (1) arises naturally as kinematic boundary condition in free boundary flows where $\mathcal{U}(\Omega)$ is given as the restriction of the velocity field of the flow to the boundary. In this context, it is simply a consequence of the demand that the change from Eulerian to Lagrangian coordinates should be a diffeomorphism.

We restrict our attention to domains that can be obtained as diffeomorphic images of a ball. This allows the following approach: Let

$$
\Phi \in C^{\omega}\left(\bar{B}_{\delta}, \mathbb{R}^{N}\right) \cap \operatorname{Diff}^{\infty}\left(B_{\delta}, \Omega_{\delta}\right)
$$

be a smooth diffeomorphism of the ball $B_{\delta}=\left\{x \in \mathbb{R}^{N}|| x \mid<1+\delta\right\}$ onto some domain $\Omega_{\delta}=\Phi\left[B_{\delta}\right] \subset \mathbb{R}^{N}, \delta>0$. It is proved in [8: Lemma 1.1] that there is a map $\Psi$ such that for all $s>\frac{N+1}{2}$

$$
\Psi \in \mathcal{L}\left(H^{s}\left(S^{N-1}\right),\left(H^{s+\frac{1}{2}}\left(B_{0}\right)\right)^{N}\right),
$$

$\Psi(r+1)=\psi_{r}$ is a (global) diffeomorphism of $B_{0}$ onto $B_{r}=\psi_{r}\left[B_{0}\right]$ if $\|r\|_{g}$ is sufficiently small, $\psi_{0}(x)=x$, and

$$
\psi_{r}(x)=(r(x)+1) x \quad \text { for all } x \in S^{N-1} .
$$

By the definition

$$
\Omega_{r}=\Phi\left[B_{r}\right]=\Phi \circ \psi_{r}\left[B_{0}\right]
$$

we introduce a one-to-one correspondence between the functions $r \in C\left(S^{N-1},(-\delta, \delta)\right)$ and certain domains whose boundaries are sufficiently close to $\Gamma_{0}=\partial \Omega_{0}$. 
In order to reformulate our domain evolution problem as an evolution equation for a function on a fixed domain of definition, we describe the moving domain by

$$
\Omega(t)=\Omega_{r(t)} .
$$

It is not hard to see that this description implies the evolution equation

$$
\dot{r}=F(\Phi, r)=\frac{\Phi^{*} U[\Phi, r] \circ \psi_{r} \cdot \nu(r)}{n \cdot \nu(r)} \quad \text { on } S^{N-1}
$$

for $r$, where

$\nu(r)=\tilde{\nu}(r) \circ \psi(r), \tilde{\nu}(r)$ denotes the outer unit normal vector field on $B_{r}$

$n=\nu(0)=\mathrm{id}_{S^{N-1}}$ is the outer normal vector field on $S^{N-1}$

$\Phi^{*}$ denotes the pull-back of a vector field by $\Phi$ from $\partial \Omega_{r}$ to $\partial B_{r}$ and

$$
U[\Phi, r]=\mathcal{U}\left(\left(\Phi \circ \psi_{r}\right)\left[S^{N-1}\right]\right)
$$

The initial condition that complements (3) is given by $\Omega(0)$.

In order to obtain an equivariance property for $F$, we choose a basis $\left\{Q_{1}, \ldots, Q_{\left(\begin{array}{c}N \\ 2\end{array}\right)}\right\}$ of the linear space of all skew-symmetric $(N, N)$-matrices and define for any $\theta \in \mathbb{R}^{\left(\begin{array}{c}N \\ 2\end{array}\right)}$ the rotation around the origin

$$
S_{\theta}=e^{\theta_{1} Q_{1}} \ldots e^{\left.\theta_{2}^{N}\right)^{Q}\left(\begin{array}{c}
N \\
2
\end{array}\right)}
$$

Lemma 1 (Equivariance of $F$ ). For any $\theta \in \mathbb{R}^{\left(\begin{array}{c}N \\ 2\end{array}\right)}$ and any $r \in C\left(S^{N-1},(-\delta, \delta)\right.$ ),

$$
F(\Phi, r) \circ S_{\theta}=F\left(\Phi \circ S_{\theta}, r \circ S_{\theta}\right) .
$$

Proof. As a consequence of the definitions and of (2),

$$
\begin{aligned}
n \circ S_{\theta} & =S_{\theta} \circ n \\
\nu(r) \circ S_{\theta} & =S_{\theta} \circ \nu\left(r \circ S_{\theta}\right) \\
\psi_{r} \circ S_{\theta} & =S_{\theta} \circ \psi_{r \circ S_{\theta}} .
\end{aligned}
$$

Hence, because of

$$
\Phi \circ \psi_{r} \circ S_{\theta}=\Phi \circ S_{\theta} \circ \psi_{r} \circ S_{\theta}
$$

we have

$$
\left(\Phi \circ \psi_{r}\right)\left[S^{N-1}\right]=\left(\Phi \circ S_{\theta} \circ \psi_{r \circ S_{\theta}}\right)\left[S^{N-1}\right]
$$

and thus

$$
U[\Phi, r]=U\left[\Phi \circ S_{\theta}, r \circ S_{\theta}\right]
$$

Consequently,

$$
\begin{aligned}
\Phi^{*} U[\Phi, r] & =T \Phi^{-1} \circ U[\Phi, r] \circ \Phi \\
& =S_{\theta} \circ S_{\theta}^{-1} \circ T \Phi^{-1} \circ U[\Phi, r] \circ \Phi \circ S_{\theta} \circ S_{\theta}^{-1} \\
& =S_{\theta} \circ T\left(\Phi \circ S_{\theta}\right)^{-1} \circ U\left[\Phi \circ S_{\theta}, r \circ S_{\theta}\right] \circ \Phi \circ S_{\theta} \circ S_{\theta}^{-1} \\
& =S_{\theta} \circ\left(\Phi \circ S_{\theta}\right)^{*} U\left[\Phi \circ S_{\theta}, r \circ S_{\theta}\right] \circ S_{\theta}^{-1} .
\end{aligned}
$$


Combining this with (5) - (7) one gets

$$
\begin{aligned}
F(\Phi, r) \circ S_{\theta} & =\frac{\Phi^{*} U[\Phi, r] \circ \psi_{r} \circ S_{\theta} \cdot \nu(r) \circ S_{\theta}}{n \circ S_{\theta} \cdot \nu(r) \circ S_{\theta}} \\
& =\frac{S_{\theta} \circ\left(\Phi \circ S_{\theta}\right)^{*} U\left[\Phi \circ S_{\theta}, r \circ S_{\theta}\right] \circ S_{\theta}^{-1} \circ S_{\theta} \circ \psi_{r \circ S_{\theta}} \cdot S_{\theta} \circ \nu\left(r \circ S_{\theta}\right)}{S_{\theta} \circ n \cdot S_{\theta} \circ \nu\left(r \circ S_{\theta}\right)} \\
& =F\left(\Phi \circ S_{\theta}, r \circ S_{\theta}\right)
\end{aligned}
$$

and the lemma is proved

The core of our problem is the investigation of the operator

$$
f: V \longrightarrow H^{s}\left(S^{N-1}\right)
$$

defined by

$$
f(\theta, r)=F\left(\Phi \circ S_{\theta}, r\right)
$$

where $V$ is a sufficiently small neighborhood of 0 in $\mathbb{R}^{\left(\frac{N}{2}\right)} \times H^{s+d}\left(S^{N-1}\right)$. The positive integer $d$ denotes the order of the considered problem.

\section{An example: Stokes flow with surface tension}

As a first step, one has to establish smoothness properties of $f$. This has to be done separately for any considered domain evolution problem, i.e. for every $\mathcal{U}$. In this section, we will give a proof for the analyticity of $f$ in the case of so-called Stokes flow driven by surface tension [18]. The methods used there can also be applied to the other evolution problems which will be discussed later. Therefore, the proof of Lemma 2 below will also serve as a model for these other applications where our attention will be restricted to the necessary changes and to new aspects.

As a preparation for the discussion of the Stokes flow problem, we introduce the bilinear operator

$$
\otimes: \mathbb{R}^{\left(\begin{array}{c}
N \\
2
\end{array}\right)} \times \mathbb{R}^{N} \longrightarrow \mathbb{R}^{N}
$$

by

$$
(a \otimes b)_{i}=\sum_{j=1}^{i-1} a_{K(j, i)} b_{j}-\sum_{j=i+1}^{N} a_{K(i, j)} b_{j} \quad(i=1, \ldots, N)
$$

where

$$
K:\{(i, j) \mid 1 \leq i<j \leq N\} \rightarrow\left\{1, \ldots,\left(\begin{array}{c}
N \\
2
\end{array}\right)\right\}
$$

is an arbitrary but fixed bijection. Moreover, we define the $\left(\begin{array}{c}N \\ 2\end{array}\right)$-vector-valued differential operator rot, acting on differentiable $N$-vector fields, by

$$
(\operatorname{rot} v)_{K(i, j)}=\partial_{i} v_{j}-\partial_{j} v_{i} \quad(1 \leq i<j \leq N) .
$$

Clearly, for $N=3$ we essentially recover the usual concepts of the vector product and the curl of a vector field. 
Let $\mathcal{U}(\Omega)$ be given as the restriction to $\partial \Omega$ of the first component of the solution $(u, p) \in\left(H^{1}(\Omega)\right)^{N} \times L^{2}(\Omega)$ to the following clliptic boundary value problem describing Stokes flow driven by surface tension forces (see, e.g., [18]):

$$
\left.\begin{array}{rlrl}
-\Delta u+\nabla p & =0 & & \text { in } \Omega \\
\operatorname{div} u & =0 & & \text { in } \Omega \\
\mathcal{T}(u, p) n & =\kappa n & & \text { on } \partial \Omega \\
\int_{\Omega} u d x & =0 & & \\
\int_{\Omega} \operatorname{rot} u d x & =0 . &
\end{array}\right\}
$$

Here $\kappa$ denotes the $((N-1)$-fold) mean curvature of $\partial \Omega$ (with the sign chosen such that $\kappa$ is negative if $\Omega$ is convex) and $\mathcal{T}(u, p)$ is the stress tensor whose coordinates are

$$
(\mathcal{T}(u, p))_{i j}=\partial_{i} u_{j}+\partial_{j} u_{i}-p \delta_{i j}
$$

It is shown in [18] that for sufficiently smooth $\Omega(8)$ is uniquely solvable, hence $\mathcal{U}$ is well-defined. More precisely, we have the following result.

Lemma 2 (Analyticity of $f$ for Stokes flow with surface tension). Let $\mathcal{U}$ be defined

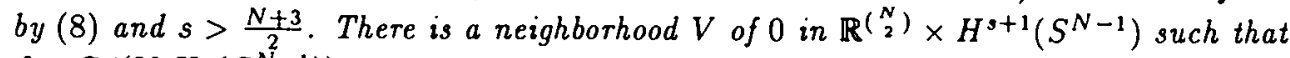
$f \in C^{\omega}\left(V, H^{s}\left(S^{N-1}\right)\right)$.

Proof. The embedding $H^{s+\frac{3}{2}}\left(B_{0}\right) \hookrightarrow C^{2}\left(B_{0}\right)$ yields that for small $r \in H^{s+1}\left(S^{N-1}\right)$ we have $\psi_{r} \in \operatorname{Diff}^{2}\left(B_{0}, B_{r}\right)$ and consequently $\Phi_{\theta, r}=\Phi \circ S_{\theta} \circ \psi_{r} \in \operatorname{Diff}^{2}\left(B_{0}, \Omega_{\theta, r}\right)$ where $\Omega_{\theta, r}=\Phi_{\theta, r}\left[B_{0}\right]$. Defining

$$
u(\theta, r)=U\left[\Phi \circ S_{\theta}, r\right] \circ \Phi_{\theta, r}
$$

we have

$$
f(\theta, r)(\xi)=\frac{S_{\theta}^{-1} T \Phi^{-1}\left(\Phi\left(S_{\theta} \psi_{r}(\xi)\right)\right) u(\theta, r)(\xi) \cdot \nu(r)(\xi)}{n \cdot \nu(r)(\xi)}
$$

and transforming (8) to $B_{0}$ by means of the diffeomorphism $\Phi_{\theta, r}$ yiclds that $u(\theta, r)$ is the first component in the solution $(u, p, \lambda)$ of the operator equation

$$
L(\theta, r)\left[\begin{array}{l}
u \\
p \\
\lambda
\end{array}\right]=\left[\begin{array}{c}
0 \\
0 \\
\kappa(\theta, r) n(\theta, r) \\
0 \\
0
\end{array}\right]
$$

$m i t h$

$$
L: V \rightarrow \mathcal{L}\left(X_{s}, Y_{s}\right)
$$

where

$$
\begin{aligned}
& X_{s}=\left(H^{s+\frac{1}{2}}\left(B_{0}\right)\right)^{N} \times H^{s-\frac{1}{2}}\left(B_{0}\right) \times\left(\mathbb{R}^{N} \times \mathbb{R}^{\left(\begin{array}{c}
N \\
2
\end{array}\right)}\right) \\
& Y_{s}=\left(H^{s-\frac{3}{2}}\left(B_{0}\right)\right)^{N} \times H^{s-\frac{1}{2}}\left(B_{0}\right) \times\left(H^{s-1}\left(S^{N-1}\right) \times \mathbb{R}^{N} \times \mathbb{R}^{\left(\begin{array}{c}
N \\
2
\end{array}\right)}\right.
\end{aligned}
$$


and $L$ is given by

$$
L(\theta, r)\left[\begin{array}{c}
u \\
p \\
\lambda
\end{array}\right]=\left[\begin{array}{c}
-\Delta_{\theta, r} u+\nabla_{\theta, r} p+\lambda_{1} \\
\operatorname{div}_{\theta, r} u \\
T_{\theta, r}(u, p) n(\theta, r)+\lambda_{2} \otimes n(\theta, r) \\
\int_{B_{0}} u \operatorname{det} \mathcal{A} d x \\
\int_{B_{0}} \operatorname{rot}_{\theta, r} u \operatorname{det} \mathcal{A} d x
\end{array}\right]
$$

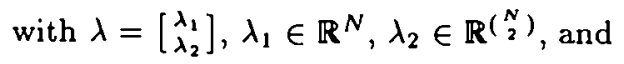

$$
\begin{aligned}
\left(\Delta_{\theta, r} u\right)_{i} & =a^{j l} \partial_{j}\left(a^{k l} \partial_{k} u_{i}\right) \\
\left(\nabla_{\theta, r} p\right)_{i} & =a^{j i} \partial_{j} p \\
\operatorname{div}_{\theta, r} u & =a^{j i} \partial_{j} u_{i} \\
\left(\mathcal{T}_{\theta, r}(u, p)\right)_{i j} & =a^{k j} \partial_{k} u_{i}+a^{k i} \partial_{k} u_{j}-p \delta_{i j} \\
\left(\operatorname{rot}_{\theta, r} u\right)_{K(i, j)} & =a^{k i} \partial_{k} u_{j}-a^{k j} \partial_{k} u_{i} \\
{[\mathcal{A}]_{i j}=[\mathcal{A}(\theta, r)]_{i j} } & =\left[D \Phi_{\theta, r}\right]_{i j}=\partial_{k} \Phi^{i} \circ S_{\theta} \circ \psi_{r} \cdot\left[S_{\theta}\right]_{k l} \cdot \partial_{j} \psi_{r}^{l} \\
a^{i j}=a^{i j}(\theta, r) & =\left[\mathcal{A}^{-1}\right]_{i j} .
\end{aligned}
$$

Moreover,

$$
\begin{aligned}
& n(\theta, r)=\tilde{n}\left[\partial \Omega_{\theta, r}\right] \circ \Phi_{\theta, r} \\
& \kappa(\theta, r)=\tilde{\kappa}\left[\partial \Omega_{\theta, r}\right] \circ \Phi_{\theta, r}
\end{aligned}
$$

where $\tilde{n}$ and $\tilde{\kappa}$ denote the operators that assign to a closed (sufficiently smooth) surface its outer normal vector and curvature, as functions valued in $\mathbb{R}^{N}$ and $\mathbb{R}$, respectively.

For any function $g \in C^{\omega}\left(\bar{B}_{\delta}\right)$, the Cauchy estimates

$$
\left\|\partial^{\alpha} g\right\|_{H^{\cdot+\frac{3}{2}\left(B_{0}\right)}} \leq C_{0, s, \delta} \alpha ! C_{1, s, \delta}^{|\alpha|}
$$

holding for all multiindices $\alpha$, together with the Banach algebra property of the space $H^{s+\frac{3}{2}}\left(B_{0}\right)$, imply analyticity of the superposition operator $u \mapsto g(u)$ from a small neighborhood of the identity in $\left(H^{s+\frac{3}{2}}\left(B_{0}\right)\right)^{N}$ into $H^{s+\frac{3}{2}}\left(B_{0}\right)$. Using this and the Banach algebra property of $H^{s+\frac{1}{2}}\left(B_{0}\right)$ as well as the analyticity of the mapping $\theta \mapsto S_{\theta}$ we find

$$
\mathcal{A} \in C^{\omega}\left(V,\left(H^{s+\frac{1}{2}}\left(B_{0}\right)\right)^{N \times N}\right) \text {. }
$$

By assumption, $\mathcal{A}(0,0)=D \Phi$ is invertible in the Banach algebra $\left(H^{s+\frac{1}{2}}\left(B_{0}\right)\right)^{N \times N}$. In any Banach algebra, the set of invertible elements is open and the inversion is a locally analytic operation, hence it follows that

$$
a^{i j} \in C^{\omega}\left(V, H^{s+\frac{1}{2}}\left(B_{0}\right)\right)
$$

Introducing arbitrary local coordinates $\left\{x_{i}\right\}$ on a subset $W \subset S^{N-1}$ having smooth -boundary one finds that $n=n(\theta, r)$ satisfies the system of equations

$$
H((\theta, r), n)=0,
$$


where

$$
H: V \times\left(H^{s}(W)\right)^{N} \longrightarrow\left(H^{s}(W)\right)^{N}
$$

is given by

$$
\begin{aligned}
H_{i}((\theta, r), n) & =\frac{\partial}{\partial x_{i}}\left(\left.\left(\Phi^{j} \circ S_{\theta} \circ \psi_{r}\right)\right|_{W}\right) n_{j} \\
& =\left.\partial_{k} \Phi^{j} \circ S_{\theta} \circ \psi_{r}\right|_{W} \cdot\left[S_{\theta}\right]_{k l} \cdot \frac{\left.\partial \psi_{r}^{l}\right|_{W}}{\partial x_{i}} \cdot n_{j} \quad(i=1, \ldots, N-1) \\
H_{N}((\theta, r), n) & =\|n\|^{2}-1 .
\end{aligned}
$$

Due to the well-known restriction theorems for Sobolev spaces and the Banach algebra property of $H^{s}(W)$ we find $H \in C^{\omega}\left(V,\left(H^{s}(W)\right)^{N}\right)$. Moreover, we clearly have

$$
\left.H\left((0,0), \tilde{n} \mid \partial \Omega_{0}\right] \circ \Phi\right)=0
$$

i.e. (11) is solvable at $(0,0)$, and the fact that $\Phi$ is a diffeomorphism implies that the Fréchet derivative of $H$ with respect to the second argument at $((0,0), n(0,0))$ is bijective. Hence it follows from the analytic version of the Implicit Function theorem (cf. [27: Chapter 8]) that there is a unique $n^{*} \in C^{\omega}\left(V,\left(H^{*}(W)\right)^{N}\right)$ satisfying (11) and (12). As (11) characterizes $n(\theta, r)$ up to the sign, we find in $W$

$$
n(\theta, r)=n^{*}(\theta, r) \quad((\theta, r) \in V) .
$$

Using partitions of unity, we conclude by standard arguments

$$
n \in C^{\omega}\left(V,\left(H^{s}\left(S^{N-1}\right)\right)^{N}\right) \text {. }
$$

Together with (10) and the Banach algebra property of the spaces $H^{s-\frac{3}{2}}\left(B_{0}\right), H^{s-\frac{1}{2}}\left(B_{0}\right)$ and $H^{s-1}\left(S^{N-1}\right)$ this implies

$$
L \in C^{\omega}\left(V, \mathcal{L}\left(X_{s}, Y_{s}\right)\right)
$$

Furthermore, on any sufficiently smooth hypersurface $\Gamma \subset \mathbb{R}^{N}$, the mean curvature $\kappa$ can be characterized by the equation

$$
\kappa n=\Delta_{\Gamma} X,
$$

where $n$ denotes again the outer normal vector field on $\Gamma$ and $\Delta_{\Gamma}$ denotes the LaplaceBeltrami operator on $\Gamma$, which has to be applied componentwise to the embedding function

$$
X: \Gamma \longrightarrow \mathbb{R}^{N}
$$

that assigns to each point of $\Gamma$ its coordinates in $\mathbb{R}^{N}$. For a proof of this see, e.g., [11: Section 2.5/Theorem 1]. Consequently, in local coordinates $\left\{x_{i}\right\}$ we have on $W$

$$
\kappa(\theta, r)=\frac{1}{\sqrt{g}} \frac{\partial}{\partial x_{i}}\left(g^{i j} \frac{\partial}{\partial x_{j}}\left(\left.\left(\Phi^{k} \circ S_{\theta} \circ \psi_{r}\right)\right|_{W}\right)\right) n_{k}(\theta, r),
$$


where

$$
\begin{aligned}
g=g(\theta, r) & =\operatorname{det} G(\theta, r) \\
{[G(\theta, r)]_{i j} } & =\frac{\partial}{\partial x_{i}}\left(\left(\Phi^{k} \circ S_{\theta} \circ \psi_{r}\right) \mid w\right) \frac{\partial}{\partial x_{j}}\left(\left(\Phi^{k} \circ S_{\theta} \circ \psi_{r}\right) \mid w\right) \\
g^{i j}=g^{i j}(\theta, r) & =\left[G(\theta, r)^{-1}\right]_{i j} .
\end{aligned}
$$

In the same way as above, using the regularity of $\Phi$, the Banach algebra property of the spaces $H^{s}\left(S^{N-1}\right)$ and $H^{s-1}\left(S^{N-1}\right)$, and the fact that the mapping $g \mapsto g^{-\frac{1}{2}}$ is analytic near any $g_{0}$ on $H^{s-1}\left(S^{N-1}\right)$ if $g_{0}$ is positive and smooth one obtains

$$
\kappa \in C^{\omega}\left(V, H^{s-1}\left(S^{N-1}\right)\right)
$$

and therefore

$$
\kappa n \in C^{\omega}\left(V, H^{s-1}\left(S^{N-1}\right)\right) .
$$

Finally, it follows from results on the regularity of (8) (see [18]) that $L(0,0)$ is a homeomorphism from $X_{s}$ onto $Y_{s}$. From this, (14), and (15) it follows by the analytic version of the Implicit Function Theorem applied to (9) that

$$
u \in C^{\omega}\left(V,\left(H^{s+\frac{1}{2}}\left(B_{0}\right)\right)^{N}\right) \text {. }
$$

The statement of the lemma follows from this by the Banach algebra property of $H^{s}\left(S^{N-1}\right)$ and the fact that

$$
\nu \in C^{w}\left(V^{\prime},\left(H^{s}\left(S^{N-1}\right)\right)^{N}\right)
$$

where $V^{\prime}$ is a sufficiently small neighbourhood of 0 in $H^{s+1}\left(S^{N-1}\right)$. Note that (16) follows from (13) by choosing $\Phi$ to be the identity and setting $\theta=0$

\section{Quasilinearization by equivariance}

The equivariance property of $F$ which was shown in Lemma 1 will enable us to prove a chain rule for the (in general nonlocal) operator $f$, i.e. an equation that links spatial derivatives of $f(\varphi, r)$ on one hand to Fréchet derivatives of $f$ applied to spatial derivatives of $r$ on the other. This chain rule will be used to identify a quasilinear structure of the operators that assign to $r$ spatial derivatives of $f(\varphi, r)$, even if the operator $r \mapsto f(\varphi, r)$ is fully nonlinear.

We do not attempt to give the most general formulation possible but one which is oriented at our applications. In this section we will assume

$$
f \in C^{K}\left(V, H^{s}\left(S^{N-1}\right)\right)
$$

with $s>s_{0}, d>0, K \in \mathbb{N}$ (or " $K=\omega$ " denoting analytic dependence), $V$ a neigh-

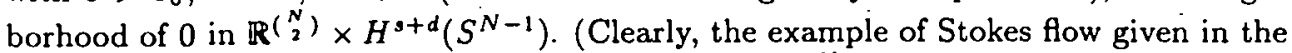
previous section fits into this frame with $K=\omega, s_{0}=\frac{N+3}{2}$, and $d=1$.) 
Consider the natural representation $U$ of the Lie group $S O(N)$ of rotations around the origin in $\mathbb{R}^{N}$ on the spaces $H^{\sigma}\left(S^{N-1}\right), \sigma \geq 0$, given by

$$
U(g) \varphi=\varphi \circ g
$$

for all $g \in S O(N)$ and all $\varphi \in H^{\sigma}\left(S^{N-1}\right)$. By standard arguments, one can show that $U$ is strongly continuous. Morcover, let us denote by $D_{j}$ the generators of the one-parameter subgroups $t \mapsto U\left(e^{-t Q_{j}}\right)$. With the usual multiindex notation, we define

$$
H^{\sigma, n}\left(S^{N-1}\right)=\bigcap_{|\alpha| \leq n} \mathcal{D}\left(D^{\alpha}\right)
$$

which is a Banach space under the norm given by

$$
\|u\|_{\sigma, n}=\sum_{|\alpha| \leq n}\left\|D^{\alpha} u\right\|_{\sigma} .
$$

It follows from [14: Theorem 3.17] that

$$
H^{\sigma, n}\left(S^{N-1}\right) \cong H^{\sigma+n}\left(S^{N-1}\right)
$$

with equivalence of the respective norms.

In the following, let $\pi_{1}$ denote the canonical projection of $\mathbb{R}^{\left(\begin{array}{c}N \\ 2\end{array}\right)} \times H^{s+d}\left(S^{N-1}\right)$ onto its first component.

Lemma 3 (A chain rule for $f$ ). Let $|\alpha|=n \leq K$ and suppose that $(\varphi, r) \in V$ and $r \in H^{s+d+n}\left(S^{N-1}\right)$. Then $f(\varphi, r) \in H^{s+n}\left(S^{N-1}\right)$ and

$$
\begin{aligned}
D^{\alpha} f(\varphi, r)= & f^{\prime}(\varphi, r)\left[\chi_{\alpha}(\varphi), D^{\alpha} r\right] \\
& +\sum_{(\underline{\beta}, k) \in \mathcal{I}_{1}} C_{\underline{\beta}, k} f^{(k)}(\varphi, r)\left[\left(\chi_{\beta_{1}}(\varphi), D^{\beta_{1}} r\right), \ldots,\left(\chi_{\beta_{k}}(\varphi), D^{\beta_{k}} r\right)\right] \\
& +\sum_{(\underline{\beta}, k, l) \in \mathcal{I}_{2}} C_{\underline{\beta}, k, l} f^{(k+l)}(\varphi, r) \\
& \times\left[\left(\chi_{\beta_{1}}(\varphi), D^{\beta_{1}} r\right), \ldots,\left(\chi_{\beta_{k}}(\varphi), D^{\beta_{k}} r\right),\left(\tilde{\chi}_{\underline{\beta}, k, l, 1}(\varphi), 0\right), \ldots,(\tilde{\chi} \underline{\beta}, k, l, l(\varphi), 0)\right]
\end{aligned}
$$

where

$$
\begin{gathered}
\mathcal{I}_{1}=\left\{(\underline{\beta}, k)|2 \leq k \leq| \alpha \mid, \underline{\beta}=\left(\beta_{1}, \ldots, \beta_{k}\right), \beta_{1}+\ldots+\beta_{k}=\alpha\right\}, \\
\mathcal{I}_{2}=\left\{(\underline{\beta}, k, l) \mid \begin{array}{l}
0 \leq k \leq|\alpha|-3,1 \leq l \leq|\alpha|-k-2 \\
\underline{\beta}=\left(\beta_{1}, \ldots, \beta_{k}\right),\left|\beta_{1}+\ldots+\beta_{k}\right| \leq|\alpha|-2
\end{array}\right\}
\end{gathered}
$$

and $\chi_{\beta}, \tilde{\chi_{\beta}, k, l, j} \in C^{\infty}\left(\left(\pi_{1}[V], \mathbb{R}^{\left(\begin{array}{c}N \\ 2\end{array}\right)}\right)\right.$.

Proof. By the Inverse Function Theorem, there is a neighborhood $W$ of 0 in $\mathbb{R}^{\left(\begin{array}{c}N \\ 2\end{array}\right)}$ such that the restriction of the mapping $\theta \mapsto S_{\theta}$ to $W$ acts as a smooth diffeomorphism 
of $W$ onto its image $W^{\prime}$. The inverse of this diffeomorphism will be denoted by $S^{-1}$. By taking $V$ sufficiently small one can arrange that $S_{\varphi} \circ S_{\theta} \in W^{\prime}$ for all $\varphi, \theta \in \pi_{1}[V]$. The mapping

$$
T:\left(\pi_{1}[V]\right)^{2} \longrightarrow W
$$

defined by

$$
T(\varphi, \theta)=S^{-1}\left(S_{\varphi} \circ S_{\theta}\right)
$$

is smooth, and for any $\varphi \in \pi_{1}[V]$ we have that $T(\varphi, \theta) \in \pi_{1}[V]$ for sufficiently small $\theta$. Hence, by Lemma 1 and the definition of $f$,

$$
f(\varphi, r) \circ S_{\theta}=f\left(T(\varphi, \theta), r \circ S_{\theta}\right)
$$

for $\theta$ sufficiently small. Differentiating this equation_with respect to $\theta$; at $\theta=0$ yields, for $r \in H^{s+d+1}\left(S^{N-1}\right)$,

$$
D_{j} f(\varphi, r)=f^{\prime}(\varphi, r)\left[\frac{\partial T}{\partial \theta_{j}}(\varphi, 0), D_{j} r\right]
$$

This equation is valid for all $j$ and the right side is in $H^{s}\left(S^{N-1}\right)$, hence $f \in H^{s, 1}\left(S^{N-1}\right)$ $=H^{s+1}\left(S^{N-1}\right)$. Thus, the lemma is proved for $|\alpha|=1$. Taking the $k$-th order Fréchet derivative $(k \leq K-1)$ on both sides of $(19)$ and writing $\frac{\partial T}{\partial \theta_{j}}(\varphi, 0)=e_{j}(\varphi)$, one finds

$$
\begin{aligned}
D_{j} f^{(k)}(\varphi, r)\left[\left(\vartheta_{1}, h_{1}\right), \ldots,\left(\vartheta_{k}, h_{k}\right)\right] \\
=f^{(k+1)}(\varphi, r)\left[\left(\vartheta_{1}, h_{1}\right), \ldots,\left(\vartheta_{k}, h_{k}\right),\left(e_{j}(\varphi), D_{j} r\right)\right] \\
\quad+\sum_{l=1}^{k} f^{(k)}(\varphi, r) \\
\quad \times\left[\left(\vartheta_{1}, h_{1}\right), \ldots,\left(\vartheta_{l-1}, h_{l-1}\right),\left(e_{j}^{\prime}(\varphi)\left[\vartheta_{l}\right], D_{j} h_{l}\right),\left(\vartheta_{l-1}, h_{l-1}\right) \ldots,\left(\vartheta_{k}, h_{k}\right)\right] \\
\quad+\sum_{(j, \underline{\nu}, \underline{\underline{1}}) \in \mathcal{I}_{3}} f^{(j)}(\varphi, r) \\
\quad \times\left[\left(\vartheta_{\nu_{1}}, h_{\nu_{1}}\right), \ldots,\left(\vartheta_{\nu_{j-1}}, h_{\nu_{j-1}}\right),\left(e_{j}^{(k-j+1)}(\varphi)\left[\vartheta_{\mu_{1}}, \ldots, \vartheta_{\mu_{k-j+1}}\right], 0\right)\right]
\end{aligned}
$$

with

$$
\mathcal{I}_{3}=\left\{(j, \underline{\nu}, \underline{\mu}) \mid \begin{array}{l}
1 \leq j \leq k-1, \underline{\nu}=\left\{\nu_{1}, \ldots, \nu_{j-1}\right\}, \underline{\mu}=\left\{\mu_{1}, \ldots, \mu_{k-j+1}\right\} \\
\underline{\nu} \cup \underline{\mu}=\{1, \ldots, k\}, \nu_{1}<\ldots<\nu_{j-1}, \mu_{1}<\ldots<\mu_{k-j+1}
\end{array}\right\}
$$

(and obvious modifications if $j=1$, i.e. $\underline{\nu}$ is empty). (20) is proved straightforwardly by induction over $k$. The proof of the lemma can be given now by induction over $|\alpha|$. Suppose the assertion holds for all multiindices $\alpha^{\prime}$ with $\left|\alpha^{\prime}\right|=n-1 \leq K-1$ and choose an $\alpha$ with $|\alpha|=n$. Then $D^{\alpha}=D_{j} D^{\alpha^{\prime}}$ for an $\alpha^{\prime}$ with $\left|\alpha^{\prime}\right|=n-1$. Applying the induction assumption and (20) yields the assertion for $\alpha$ 

problem

Clearly, our interest is in the evolution (3) for fixed $\Phi$, i.e. in the nonlinear Cauchy

$$
\left.\begin{array}{rl}
\dot{r} & =\rho(r)=f(0, r) \\
r(0) & =r_{0} .
\end{array}\right\}
$$

Assumption (17) and Lemma 3 yield the following key results on the operator $\rho$. that:

Lemma 4 (Propertics of $\rho$ ). There is a neighborhood $\mathcal{W}$ of 0 in $H^{s}\left(S^{N-1}\right)$ such

(i) (Regularity).

$$
\rho \in C^{K-n}\left(\mathcal{W} \cap H^{s+d+n}\left(S^{n-1}\right), H^{s+n}\left(S^{N-1}\right)\right)
$$

and $\rho$ is bounded from $\mathcal{W} \cap H^{s+d+n}\left(S^{N-1}\right)$ to $H^{s+n}\left(S^{N-1}\right)$ for all non-negative integers $n \leq K$.

(ii) (Analyticity). If $K=\omega$, then

$$
\rho \in C^{\omega}\left(\mathcal{W} \cap H^{s+d+n}\left(S^{N-1}\right), H^{s+n}\left(S^{N-1}\right)\right)
$$

for all $n \in \mathbb{N}$.

(iii) (Weak sequential continuity). For all non-negative integers $n \leq K, \rho$ is weakly sequentially continuous from $\mathcal{W} \cap H^{s+d+n}\left(S^{N-1}\right)$ into $H^{s+n}\left(S^{N-1}\right)$.

(iv) (Quasilinearity of $D^{\alpha} \circ \rho$ ). For any multiindex $\alpha$ with $0<|\alpha| \leq n \leq K$ there is an operator $G_{\alpha} \in C^{K-n}\left(\mathcal{W} \cap H^{s+n+d-1}\left(S^{N-1}\right), H^{s}\left(S^{N-1}\right)\right)$ such that

$$
D^{\alpha} \rho(r)=\rho^{\prime}(r)\left[D^{\alpha} r\right]+G_{\alpha}(r)
$$

satisfying for all $r \in \mathcal{W} \cap H^{s+d+n}\left(S^{N-1}\right)$ and arbitrary $\varepsilon>0$ the estimates

$$
\begin{aligned}
& \left\|G_{\alpha}(r)\right\|_{s} \leq C_{s, n}\left(\|r\|_{s+n+d-1}^{2}+1\right) \\
& \left\|G_{\alpha}(r)\right\|_{s} \leq \varepsilon\|r\|_{s+n}+g_{s, n, \varepsilon}\left(\|r\|_{s+d+\frac{1}{2}}\right)
\end{aligned}
$$

where $g_{s, n, \varepsilon}: \mathbb{R}_{+} \longrightarrow \mathbb{R}_{+}$is a monotone increasing function.

Proof. The smoothness results in (i) and (ii) are immediate consequences of the chain rule in Lemma 3 with $\varphi=0$ and the norm equivalence (18). The same is true for the boundedness result if $K$ is finite. For $K=\omega$, the boundedness of $\rho$ follows from the fact that (17) implies the boundedness of all Fréchet derivatives $f^{(k)}(0, r)$ on $\pi_{1}[V]$ with values in $\mathcal{L}\left(\left(H^{s+d}\left(S^{N-1}\right)\right)^{k}, H^{s}\left(S^{N-1}\right)\right)$.

(iii) follows by standard arguments from the boundedness of $\rho$ and the compactness of the embeddings $H^{\sigma}\left(S^{N-1}\right) \hookrightarrow H^{s}\left(S^{N-1}\right)$ for $\sigma>s$ (cf., e.g., [18: Proof of Lemma $7 /($ ii)]).

To show (iv), we use that

$$
f^{\prime}(0, r)\left[\left(\chi_{\alpha}(0), D^{\alpha} r\right)\right]=\rho^{\prime}(r)\left[D^{\alpha} r\right]+f^{\prime}(0, r)\left[\left(\chi_{\alpha}(0), 0\right)\right]
$$


Thus, we find (22) with

$$
\begin{aligned}
G_{\alpha}(r)= & f^{\prime}(0, r)\left[\left(\chi_{\alpha}(0), 0\right)\right] \\
& +\sum_{(\underline{\beta}, k) \in \mathcal{I}_{1}} C_{\underline{\beta}, k} f^{(k)}(0, r)\left[\left(\chi_{\beta_{1}}(0), D^{\beta_{1}} r\right), \ldots,\left(\chi_{\beta_{k}}(0), D^{\beta_{k}} r\right)\right] \\
& +\sum_{(\underline{\beta}, k, l) \in \mathcal{I}_{2}} C_{\underline{\beta}, k, l} f^{(k+l)}(0, r) \\
& \times\left[\left(\chi \beta_{\beta_{1}}(0), D^{\beta_{1}} r\right), \ldots,\left(\chi_{\beta_{k}}(0), D^{\beta_{k}} r\right),(\tilde{\chi} \underline{\underline{\beta}, k, l, 1}(0), 0), \ldots,(\tilde{\chi} \underline{\underline{\beta}, k, l, l}(0), 0)\right],
\end{aligned}
$$

and the definition of $\mathcal{I}_{1}$ and $\mathcal{I}_{2}$ as given in Lemma 3. Note that $G_{\alpha}(r)$ can be expanded in a sum of terms whose $H^{s}\left(S^{N-1}\right)$-norm can be estimated either by a constant $C_{n}$ or by $C_{n}\|r\|_{s+d+n-1}$ or by a product of the form

$$
C_{n}\|r\|_{s+d+b_{1}} \cdots\|r\|_{9+d+b_{k}}
$$

with $k \geq 2,1 \leq b_{j} \leq n-1$ and $\sum_{j=1}^{k} b_{j} \leq n$. Estimate (23) follows from this by applying the interpolation inequalities

$$
\|r\|_{s+d+b_{j}} \leq C_{s}\|r\|_{s+d+n-1}^{\frac{b_{j}}{b_{1}+\ldots+b_{k}-1}}\|r\|_{s+d}^{1-\frac{b_{j}}{b_{1}+\ldots+b_{k-1}}}
$$

for $j=1, \ldots, k-1$. To show (24) we note that there is a $\zeta>0$ depending only on $n$ such that

$$
s+d+b_{j} \leq(s+d+n-\zeta) \frac{b_{j}}{n}+\left(s+d+\frac{1}{2}\right)\left(1-\frac{b_{j}}{n}\right)
$$

hence

$$
\|r\|_{s+d+b_{j}} \leq C_{s}\|r\|_{s+d+n-\zeta}^{\frac{b_{j}}{n}}\|r\|_{s+d+\frac{1}{2}}^{1-\frac{b_{j}}{n}} .
$$

The estimate follows from this by application of the interpolation inequality

$$
\|r\|_{s+d+n-\zeta} \leq \varepsilon^{\prime}\|r\|_{s+d+n}^{\prime}+C_{\epsilon^{\prime}}\|r\|_{s+d}
$$

with $\varepsilon^{\prime}>0$ sufficiently small

\section{Linearization}

It is clear from the results of the previous section that the study of the linear operator $\rho^{\prime}(r)$ is essential for the analysis of equation (3). At first, we will discuss a "change of coordinates" for our problem.

For the description of the linearized evolution problem, it is more appropriate to choose $\Gamma_{0}=\Phi_{0}\left(S^{N-1}\right)$ as fixed reference manifold and to consider small perturbations of $\Gamma_{0}$ in normal direction. This approach leads to the following additional definitions: There is a neighborhood $\tilde{U}$ of $\Gamma_{0}$ in $\mathbb{R}^{N}$ such that there are a $\bar{\delta}>0$ and a smooth diffeomorphism

$$
\Theta: \Gamma_{0} \times(-\bar{\delta}, \bar{\delta}) \longrightarrow \tilde{U}
$$


given by

$$
\Theta(\bar{\xi}, \eta)=\bar{\xi}+\eta \bar{n}(\bar{\xi})
$$

where $\bar{n}$ denotes the outer normal vector field on $\Gamma_{0}$. We denote the inverse of $\Theta$ by $\Theta^{-1}=(\Xi, d)$. It is straightforward to check that for smooth $r$ with $\|r\|_{C^{1}\left(S^{N-1}\right)}$ sufficiently small, there is precisely one function $R \in C^{\infty}\left(\Gamma_{0}\right)$ defined by

$$
R(r)\left(\Xi \Phi \psi_{r}(\xi)\right)=d\left(\Phi \psi_{r}(\xi)\right) \quad\left(\xi \in S^{N-1}\right)
$$

and that $R(r)$ depends smoothly on $r$. Note that $R(r)$ describes the perturbed domain $\Omega_{r}$ with respect to the coordinates in $\tilde{U}$ given by $\Theta$, i.e.

$$
\partial \Omega_{r}=\left\{\Theta(\bar{\xi}, R(r)(\bar{\xi})) \mid \bar{\xi} \in \Gamma_{0}\right\}
$$

The evolution (3) can therefore be described equivalently by

$$
\dot{R}=\bar{\rho}(R)
$$

and it is our aim to express $\rho^{\prime}(r)$ with the help of $\bar{\rho}^{\prime}(R(r))$ which can be calculated more straightforwardly.

The relation between $\rho$ and $\bar{\rho}$ is given by carrying out the differentiation of $R(r)$ with respect to $t$ which yields

$$
\bar{\rho}(R(r))=R^{\prime}(r)[\dot{r}]=R^{\prime}(r)[\rho(r)] .
$$

Calculation of the Fréchet derivatives on both sides gives

$$
R^{\prime}(r)\left[\rho^{\prime}(r)[h]\right]+R^{\prime \prime}(r)[h, \rho(r)]=\bar{\rho}^{\prime}(R(r))\left[R^{\prime}(r)[h]\right],
$$

and it remains to calculate the Fréchet derivatives $R^{\prime}(r)$ and $R^{\prime \prime}(r)$. This can be done by differentiating both sides of (25) with the result (in arbitrary local coordinates)

$$
\begin{aligned}
& R^{\prime}(r)[h]\left(\Xi \Phi \psi_{r}(\xi)\right) \\
& =a^{j}(\xi, r(\xi)) \frac{R(r)}{\bar{\xi}^{j}}\left(\Xi \Phi \psi_{r}(\xi)\right) h(\xi)+\bar{n}\left(\Xi \Phi \psi_{r}(\xi)\right) \cdot D \Phi\left(\left(\psi_{r}(\xi)\right) n(\xi) h(\xi)\right. \\
& \quad=a^{j}(\xi, r(\xi)) \frac{R(r)}{\bar{\xi}^{j}}\left(\Xi \Phi \psi_{r}(\xi)\right) h(\xi)+\gamma(\xi, r(\xi)) h(\xi),
\end{aligned}
$$

where $a^{j}, \gamma \in C^{\infty}\left(S^{N-1} \times(-\delta, \delta)\right)$. As $\Phi$ is a diffeomorphism, we have

$$
\gamma(\xi, 0)=\bar{n}(\Phi(\xi)) \cdot D \Phi(\xi) n(\xi)>\gamma_{0}>0 \quad\left(\xi \in S^{N-1}\right) .
$$

Further differentiation of (27) yields

$$
\begin{aligned}
R^{\prime \prime}(r)[h, k]\left(\Xi \Phi \psi_{r}(\xi)\right)= & a^{j}(\xi, r(\xi)) \frac{\partial}{\partial \bar{\xi}^{j}}\left(R^{\prime}(r)[h]\right)\left(\Xi \Phi \psi_{r}(\xi)\right) k(\xi) \\
& +a^{j}(\xi, r(\xi)) \frac{\partial}{\partial \bar{\xi}^{j}}\left(R^{\prime}(r)[k]\right)\left(\Xi \Phi \psi_{r}(\xi)\right) h(\xi) \\
& +\frac{\partial}{\partial r} a^{j}(\xi, r(\xi)) \frac{\partial R(r)}{\partial \bar{\xi}^{j}}\left(\Xi \Phi \psi_{r}(\xi)\right) h(\xi) k(\xi) \\
& -a^{j}(\xi, r(\xi)) a^{k}(\xi, r(\xi)) \frac{\partial^{2} R(r)}{\partial \bar{\xi}^{j} \partial \bar{\xi}^{k}}\left(\Xi \Phi \psi_{r}(\xi)\right) h(\xi) k(\xi) \\
& +\frac{\partial \gamma}{\partial r}(\xi, r(\xi)) h(\xi) k(\xi) .
\end{aligned}
$$


Combining this with (26) and (27) we find for $r=0$

$$
\rho^{\prime}(0)[h]=\frac{1}{\gamma} \phi^{*} \bar{\rho}^{\prime}(0)\left[\phi^{*-1} \gamma h\right]+l[h]+\chi h,
$$

where $\phi=\left.\Phi\right|_{S^{N-1}} \in \operatorname{Diff}^{\infty}\left(S^{N-1}, \Gamma_{0}\right), l$ is a first-order differential operator on $S^{N-1}$ corresponding to a smooth vector field and $\chi \in C^{\infty}\left(S^{N-1}\right)$. Based on (28) we show that under suitable assumptions the coercivity of $\bar{\rho}^{\prime}(0)$ implies coercivity of $\rho^{\prime}(0)$. For this purpose, we specify the scalar products which are used on $H^{s}\left(S^{N-1}\right)$ as

$$
(u, v)_{0}=\int_{S^{N-1}} u v d \omega \quad \text { and } \quad(u, v)_{\sigma}=\left(\Lambda^{\sigma} u, \Lambda^{\sigma} v\right)_{0}
$$

where

$$
\Lambda^{\sigma}=\left(I-\Delta_{S^{N-1}}\right)^{\frac{\sigma}{2}} \quad(\sigma \in \mathbb{R}),
$$

and $\Delta_{S^{N-1}}$ is the Laplace-Beltrami operator on $S^{N-1}$. It is well-known that $\Lambda^{\sigma}$ is a well-defined, $L^{2}$-self-adjoint pseudodifferential operator of order $\sigma$ on $S^{N-1}$ which maps real-valued functions to real-valued functions and that the scalar products defined above generate the usual topology on $H^{\sigma}\left(S^{N-1}\right)$. Consequently,

$$
\Lambda^{\sigma} \in \mathcal{L}_{i s}\left(H^{t}\left(S^{N-1}\right), H^{t-\sigma}\left(S^{N-1}\right)\right) \quad(t \in \mathbb{R}) .
$$

In an analogous way, scalar products $(\cdot, \cdot)^{\Gamma_{0}}$ are defined on the spaces $H^{\sigma}\left(\Gamma_{0}\right)$, using the operators $\Lambda_{\Gamma_{0}}^{o}=\left(I-\Delta_{\Gamma_{0}}\right)^{\frac{a}{2}}$ that have analogous properties.

Lemma 5 (Coercivity of $\rho^{\prime}(0)$ ). Let $s \in \mathbb{R}$ be fixed. Suppose that $\vec{\rho}^{\prime}(0)=A+K$ where $A$ is a pseudodifferential operator of order $d>0$ and

$$
K \in \mathcal{L}\left(H^{s+\frac{d-1}{2}}\left(\Gamma_{0}\right), H^{s-\frac{d-1}{2}}\left(\Gamma_{0}\right)\right) .
$$

If $A$ satisfies

$$
(A u, u)_{s}^{\Gamma_{0}} \leq-c\|u\|_{s+\frac{d}{2}}^{\Gamma_{0}}{ }^{2}+C\|u\|_{s}^{\Gamma_{0}^{2}} \quad\left(u \in H^{s+d}\left(\Gamma_{0}\right)\right)
$$

with constants $c>0$ and $C>0$, then there are (different) constants $c>0$ and $C>0$ such that

$$
\left(\rho^{\prime}(0)[h], h\right)_{s} \leq-c\|h\|_{s+\frac{d}{2}}^{2}+C\|h\|_{s}^{2} \quad\left(h \in H^{s+d}\left(S^{N-1}\right)\right) .
$$

Remark. It is clear that assumption (29) is equivalent to an analogous inequality for $\bar{\rho}^{\prime}(0)$.

Proof of Lemma 5. We have

$$
\left(\rho^{\prime}(0)[h], h\right)_{s}=\left(\frac{1}{\gamma} \phi^{*} A \phi^{*-1} \gamma h, h\right)_{s}+\left(\frac{1}{\gamma} \phi^{*} K \phi^{*-1} \gamma h, h\right)_{s}+(l[h], h)_{s}+(\chi h, h)_{s}
$$

and estimate the terms on the right separately. 
As $h \mapsto \chi h \in \mathcal{L}\left(H^{s}\left(S^{N-1}\right)\right)$ we have $(\chi h, h)_{s} \leq C\|h\|_{s}^{2}$. Note that $\left(l+l^{*}\right) h=\theta h$, where $l^{*}$ denote the $L^{2}$-adjoint of $l$ and $\theta \in C^{\infty}\left(S^{N-1}\right)$, hence $l+l^{*} \in \mathcal{L}\left(L^{2}\left(S^{N-1}\right)\right)$ and $\Lambda^{s} l-l \Lambda^{s}$ is a pseudodifferential operator of order $s$ on $S^{N-1}$, hence $\Lambda^{s} l-l \Lambda^{s} \in$ $\mathcal{L}\left(H^{s}\left(S^{N-1}\right), L^{2}\left(S^{N-1}\right)\right)$. Thus

$$
\begin{aligned}
(l[h], h)_{s} & =\left(\Lambda^{s} l[h], \Lambda^{s} h\right)_{0} \\
& =\left(l \Lambda^{s} h, \Lambda^{s} h\right)_{0}+\left(\left(\Lambda^{s} l-l \Lambda^{s}\right) h, \Lambda^{s} h\right)_{0} \\
& =\frac{1}{2}\left(\left(l+l^{*}\right) h, \Lambda^{s} h\right)_{0}+\left(\left(\Lambda^{s} l-l \Lambda^{s}\right) h, \Lambda^{s} h\right)_{0} \\
& \leq C\|h\|_{s}^{2} .
\end{aligned}
$$

As $\gamma$ is smooth and positive and $\phi$ is a smooth diffeomorphism, we have $h \mapsto \gamma h \epsilon$ $\mathcal{L}_{i s}\left(H^{s+\frac{d-1}{2}}\left(S^{N-1}\right)\right)$ and $\phi^{*} \in \mathcal{L}_{i s}\left(H^{s+\frac{d-1}{2}}\left(\Gamma_{0}\right), H^{s+\frac{d-1}{2}}\left(S^{N-1}\right)\right)$. This implies, by an interpolation inequality,

$$
\begin{aligned}
\left(\frac{1}{\gamma} \phi^{*} K \phi^{*-1} \gamma h, h\right)_{s} & =\left(\Lambda^{-\frac{d-1}{2}} \frac{1}{\gamma} \phi^{*} K \phi^{*-1} \gamma h, \Lambda^{\frac{d-1}{2}} h\right)_{s} \\
& \leq C\|h\|_{s+\frac{d-1}{2}}^{2} \\
& \leq \varepsilon\|h\|_{s+\frac{d}{2}}^{2}+C_{e}\|h\|_{s}^{2}
\end{aligned}
$$

with arbitrary $\varepsilon>0$.

By a transformation of the integration variable, we have

$$
(u, v)_{0}=\left(\mu^{\frac{1}{2}} \phi^{*-1} u, \mu^{\frac{1}{2}} \phi^{*-1} v\right)_{0}^{\Gamma_{0}}
$$

where $\mu$ is a smooth positive function on $\Gamma_{0}$ representing the "change of the surface element". Using this, one calculates

$$
\begin{aligned}
&\left(\frac{1}{\gamma} \phi^{*} A \phi^{*-1} \gamma h, h\right)_{s} \\
&=\left(\left(\frac{1}{\gamma} \phi^{*} A \phi^{*-1} \gamma I-\phi^{*} A \phi^{*-1}\right) h, h\right)_{s} \\
&+\left(\left(\Lambda^{s} \phi^{*} A \phi^{*-1}-\phi^{*} A \phi^{*-1} \Lambda^{s}\right) h, \Lambda^{s} h\right)_{0} \\
&+\left(\left(\mu^{\frac{1}{2}} A-A \mu^{\frac{1}{2}}\right) \phi^{*-1} \Lambda^{s} h, \mu^{\frac{1}{2}} \phi^{*-1} \Lambda^{s} h\right)_{0}^{\Gamma_{0}} \\
&+\left(\left(\Lambda_{\Gamma_{0}}^{-s} A-A \Lambda_{\Gamma_{0}}^{-s}\right) \mu^{\frac{1}{2}} \phi^{*-1} \Lambda^{s} h, \Lambda_{\Gamma_{0}}^{-s} \mu^{\frac{1}{2}} \phi^{*-1} \Lambda^{s} h\right)_{s}^{\Gamma_{0}} \\
&+\left(A \Lambda_{\Gamma_{0}}^{-s} \mu^{\frac{1}{2}} \phi^{*-1} \Lambda^{s} h, \Lambda_{\Gamma_{0}}^{-s} \mu^{\frac{1}{2}} \phi^{*-1} \Lambda^{s} h\right) \\
&=\left(\Lambda^{-\frac{d-1}{2}}\left(\frac{1}{\gamma} \phi^{*} A \phi^{*-1} \gamma I-\phi^{*} A \phi^{*-1}\right) h, \Lambda^{\frac{d-1}{2}} h\right)_{s} \\
&+\left(\Lambda^{-\frac{d-1}{2}}\left(\Lambda^{s} \phi^{*} A \phi^{*-1}-\phi^{*} A \phi^{*-1} \Lambda^{s}\right) h, \Lambda^{s+\frac{d-1}{2}} h\right)_{0}
\end{aligned}
$$




$$
\begin{aligned}
& +\left(\Lambda_{\Gamma_{0}}^{-\frac{d-1}{2}}\left(\mu^{\frac{1}{2}} A-A \mu^{\frac{1}{2}}\right) \phi^{*-1} \Lambda^{s} h, \Lambda_{\Gamma_{0}}^{\frac{d-1}{2}} \mu^{\frac{1}{2}} \phi^{*-1} \Lambda^{s} h\right)_{0}^{\Gamma_{0}} \\
& +\left(\Lambda_{\Gamma_{0}}^{-\frac{d-1}{2}}\left(\Lambda_{\Gamma_{0}}^{-s} A-A \Lambda_{\Gamma_{0}}^{-s}\right) \mu^{\frac{1}{2}} \phi^{*-1} \Lambda^{s} h, \Lambda_{\Gamma_{0}}^{-s+\frac{\delta-1}{2}} \mu^{\frac{1}{2}} \phi^{*-1} \Lambda^{s} h\right)_{s}^{\Gamma_{0}} \\
& +\left(A \Lambda_{\Gamma_{0}}^{-s} \mu^{\frac{1}{2}} \phi^{*-1} \Lambda^{s} h, \Lambda_{\Gamma_{0}}^{-s} \mu^{\frac{1}{2}} \phi^{*-1} \Lambda^{s} h\right) \\
& \leq-c\left\|\Lambda_{\Gamma_{0}}^{-s} \mu^{\frac{1}{2}} \phi^{*-1} \Lambda^{s} h\right\|_{s+\frac{d}{2}}^{2}+C\left\|\Lambda_{\Gamma_{0}}^{-s} \mu^{\frac{1}{2}} \phi^{*-1} \Lambda^{s} h\right\|_{s}^{2}+C\|h\|_{s+\frac{d-1}{2}}^{2} \\
& \leq-c\|h\|_{s+\frac{d}{2}}^{2}+C\|h\|_{s}^{2},
\end{aligned}
$$

where we have used that, due to the commutator properties of pseudodifferential operators,

$$
\begin{aligned}
\frac{1}{\gamma} \phi^{*} A \phi^{*-1} \gamma I-\phi^{*} A \phi^{*-1} & \in \mathcal{L}\left(H^{s+\frac{d-1}{2}}\left(S^{N-1}\right), H^{s-\frac{d-1}{2}}\left(S^{N-1}\right)\right) \\
\Lambda^{s} \phi^{*} A \phi^{*-1}-\phi^{*} A \phi^{*-1} \Lambda^{s} & \in \mathcal{L}\left(H^{s+\frac{d-1}{2}}\left(S^{N-1}\right), H^{-\frac{d-1}{2}}\left(S^{N-1}\right)\right) \\
\mu^{\frac{1}{2}} A-A \mu^{\frac{1}{2}} & \in \mathcal{L}\left(H^{\frac{d-1}{2}}\left(\Gamma_{0}\right), H^{-\frac{d-1}{2}}\left(\Gamma_{0}\right)\right) \\
\Lambda_{\Gamma_{0}}^{-s} A-A \Lambda_{\Gamma_{0}}^{-s} & \in \mathcal{L}\left(H^{\frac{d-1}{2}}\left(\Gamma_{0}\right), H^{s-\frac{d-1}{2}}\left(\Gamma_{0}\right)\right)
\end{aligned}
$$

furthermore

$$
\Lambda_{\Gamma_{0}}^{-s} \mu^{\frac{1}{2}} \phi^{*-1} \Lambda^{s} \in \mathcal{L}_{i s}\left(H^{s+\frac{d}{2}}\left(S^{N-1}\right), H^{s+\frac{d}{2}}\left(\Gamma_{0}\right)\right),
$$

and the interpolation inequality

$$
\|h\|_{9+\frac{d-1}{2}} \leq \tilde{\varepsilon}\|h\|_{9+\frac{d}{2}}^{2}+C_{\tilde{\varepsilon}}\|h\|_{s}^{2}
$$

with sufficiently small $\tilde{\varepsilon}$. The assertion follows by combining the above inequalities and choosing $\varepsilon$ in (31) sufficiently small

The following abstract lemma provides a general result concerning generator properties of a linear operator satisfying a coercivity estimate in a scale of (real) Hilbert spaces.

Lemma 6 (Generator property). For $s \in \mathbb{R}$ and $d>0$ let

$$
\begin{aligned}
A \in & \mathcal{L}\left(H^{s+\frac{3}{2} d}\left(S^{N-1}\right), H^{s+\frac{d}{2}}\left(S^{N-1}\right)\right) \\
& \cap \mathcal{L}\left(H^{s+d}\left(S^{N-1}\right), H^{s}\left(S^{N-1}\right)\right) \\
& \cap \mathcal{L}\left(H^{s+\frac{d}{2}}\left(S^{N-1}\right), H^{s-\frac{d}{2}}\left(S^{N-1}\right)\right)
\end{aligned}
$$

satisfy estimates

$$
\begin{aligned}
(A u, u)_{s+\frac{d}{2}} & \geq c\|u\|_{s+d}^{2}-C\|u\|_{s+\frac{d}{2}}^{2} & & \left(u \in H^{s+\frac{3}{2} d}\left(S^{N-1}\right)\right) \\
(A u, u)_{s} & \geq c\|u\|_{s+\frac{d}{2}}^{2}-C\|u\|_{s}^{2} & & \left(u \in H^{s+d}\left(S^{N-1}\right)\right)
\end{aligned}
$$

with constants $c>0$ and $C>0$. Then $A \in \mathcal{H}\left(H^{s+d}\left(S^{N-1}\right), H^{s}\left(S^{N-1}\right)\right)$, i.e. $-A$, considered as an unbounded operator on $H^{s}\left(S^{N-1}\right)$ with $\mathcal{D}(-A)=H^{s+d}\left(S^{N-1}\right)$, is the generator of a holomorphic semigroup on $H^{s}\left(S^{N-1}\right)$. 
Proof. We fix an arbitrary real $\omega \geq C$, define $\tilde{A}=A+\omega I$, and show below that

(i) $\tilde{A} \in \mathcal{L}_{i s}\left(H^{s+d}\left(S^{N-1}\right), H^{s}\left(S^{N-1}\right)\right)$

(ii) $\operatorname{Re}[\tilde{A} z, z]_{s} \geq 0$ for all $z \in H^{s+d}\left(S^{N-1}\right)_{\mathbb{C}}$ and $\left|\operatorname{Im}[\tilde{A} z, z]_{s}\right| \leq C \operatorname{Re}[\tilde{A} z, z]_{s}$ for all $z \in H^{s+d}\left(S^{N-1}\right) \mathrm{c}$

where $[\cdot, \cdot]_{g}$ denotes the usual scalar product in the complexified space $H^{s}\left(S^{N-1}\right) \mathbf{C}$ to which $\tilde{A}$ is extended in the natural way. As (i) holds for all real $\omega>C$, we have that $\{\lambda \in \mathbb{R} \mid \lambda \leq 0\} \subset \rho(\tilde{A})$ for any such $\omega$. The estimates (ii) mean that the numerical range of $\tilde{A}$ is contained in a sector of the right half complex plane whose opening angle is smaller than $\pi$. By [24: Proposition VII.3.2] this implies $\tilde{A} \in \mathcal{H}\left(H^{s+d}\left(S^{N-1}\right), H^{s}\left(S^{N-1}\right)\right)$ and therefore by a well-known perturbation result also $A=\tilde{A}-\omega I \in \mathcal{H}\left(H^{s+d}\left(S^{N-1}\right), H^{s}\left(S^{N-1}\right)\right)$.

To show assertion (i), consider the operator

$$
J: H^{s}\left(S^{N-1}\right) \longrightarrow\left(H^{s+d}\left(S^{N-1}\right)\right)^{\prime}
$$

given by

$$
\langle J u, v\rangle=\left(\Lambda^{-\frac{d}{2}} u, \Lambda^{\frac{d}{2}} v\right)_{s+\frac{d}{2}}
$$

which is easily shown to be an isometric isomorphism. Our assumptions imply $J \tilde{A} \in$ $\mathcal{L}\left(H^{s+d}\left(S^{N-1}\right),\left(H^{s+d}\left(S^{N-1}\right)\right)^{\prime}\right)$ and

$$
\langle J \tilde{A} u, u\rangle=(\tilde{A} u, u)_{s+\frac{d}{2}} \geq c\|u\|_{s+d}^{2} \quad\left(u \in H^{s+\frac{3}{2} d}\left(S^{N-1}\right)\right) .
$$

Hence, by a density argument,

$$
\langle J \tilde{A} u, u\rangle \geq c\|u\|_{s+d}^{2} \quad\left(u \in H^{s+d}\left(S^{N-1}\right)\right)
$$

and thus $J \tilde{A} \in \mathcal{L}_{i s}\left(H^{s+d}\left(S^{N-1}\right),\left(H^{s+d}\left(S^{N-1}\right)\right)^{\prime}\right)$ by the Lax-Milgram lemma. This implies assertion (i).

To show assertion (ii), we estimate for arbitrary $z=u+i v \in H^{s+d}\left(S^{N-1}\right)_{C}$ with $u, v \in H^{s+d}\left(S^{N-1}\right)$,

$$
\operatorname{Re}[\tilde{A} z, z]_{s}=(\tilde{A} u, u)_{s}+(\tilde{A} v, v)_{s} \geq c\left(\|u\|_{s+\frac{d}{2}}^{2}+\|v\|_{s+\frac{d}{2}}^{2}\right) \geq 0
$$

and

$$
\begin{aligned}
\left|\operatorname{Im}[\tilde{A} z, z]_{s}\right| & \leq\left|(\tilde{A} u, v)_{s}\right|+\left|(\tilde{A} v, u)_{s}\right| \\
& \leq\|\tilde{A} u\|_{s-\frac{d}{2}}\|v\|_{s+\frac{d}{2}}+\|\tilde{A} v\|_{s-\frac{d}{2}}\|u\|_{s+\frac{d}{2}} \\
& \leq C\|u\|_{s+\frac{d}{2}}\|v\|_{s+\frac{d}{2}} \\
& \leq C\left(\|u\|_{s+\frac{d}{2}}^{2}+\|v\|_{s+\frac{d}{2}}^{2}\right) \\
& \leq C \operatorname{Rc}[\tilde{A} z, z]_{s}
\end{aligned}
$$

and the lemma is proved 


\section{Solvability of the nonlinear problem}

We will show short-time solvability of the evolution problem (21) using a rather classical method of a priori energy estimates for adapted scalar products $(\cdot, \cdot)_{s, n}$ given by

$$
(u, v)_{s, n}=\sum_{|\alpha| \leq n}\left(D^{\alpha} u, D^{\alpha} v\right)_{s}
$$

Note that the norm $\|\cdot\|_{s, n}$ given by

$$
\|u\|_{s, n}^{2}=(u, u)_{s, n}
$$

is a norm on $H^{s+n}\left(S^{N-1}\right)$ that generates the usual topology (cf. (18)). Throughout this section, we assume $d \geq 1$ and $K=\omega$. (If $K$ is finite, then the results obtained below hold only for $n \leq K$.)

Lemma 7 (Local a priori estimate).

(i) (Parabolic equations): Suppose (17) with $s$ replaced by $s-\frac{d}{2}$ and (30) hold. Then for all $n \geq d$

$$
(\rho(r), r)_{s, n} \leq C_{s, n}\left(\|r\|_{s, n}^{2}+1\right) \quad\left(r \in \mathcal{W} \cap H^{s+d+n}\left(S^{N-1}\right)\right)
$$

(ii) (First-order hyperbolic equations): Suppose $d=1$, (17), and

$$
\left(\rho^{\prime}(r)[h], h\right)_{s} \leq C_{s}\|h\|_{s}^{2} \quad\left(r \in \mathcal{W}, h \in H^{s+1}\left(S^{N-1}\right)\right) .
$$

Then for all $n \geq 1$

$$
(\rho(r), r)_{s, n} \leq C_{s, n}\left(\|r\|_{s, n}^{3}+1\right) \quad\left(r \in \mathcal{W} \cap H^{s+n+1}\left(S^{N-1}\right)\right) .
$$

Proof. Using (22), we decompose

$$
\begin{aligned}
(\rho(r), r)_{s, n} & =\sum_{|\alpha| \leq n}\left(D^{\alpha} \rho(r), D^{\alpha} r\right)_{s} \\
& =(\rho(r), r)_{s}+\sum_{1 \leq|\alpha| \leq n}\left(\left(\rho^{\prime}(r)\left[D^{\alpha} r\right], D^{\alpha} r_{,}\right)_{s}+\left(G_{\alpha}(r), D^{\alpha} r\right)_{s}\right)
\end{aligned}
$$

and estimate the terms on the right separately.

To show (i), note that due to (30) for all $r \in \mathcal{W}$ and all $h \in H^{s+d}\left(S^{N-1}\right)$

$$
\begin{aligned}
\left(\rho^{\prime}(r)[h], h\right)_{s} & =\left(\rho^{\prime}(0)[h], h\right)_{s}+\left(\left(\rho^{\prime}(r)-\rho^{\prime}(0)\right)[h], h\right)_{s} \\
& \leq-c\|h\|_{s+\frac{d}{2}}^{2}+C\|h\|_{s}^{2}+\left\|\left(\rho^{\prime}(r)-\rho^{\prime}(0)\right)[h]\right\|_{s-\frac{d}{2}}\|h\|_{s+\frac{d}{2}} \\
& \leq-c^{\prime}\|h\|_{s+\frac{d}{2}}^{2}+C\|h\|_{s}^{2}
\end{aligned}
$$


if $\mathcal{W}$ is chosen sufficiently small. Consequently, using (24) with $s$ replaced by $s-\frac{d}{2}$,

$$
\begin{aligned}
(\rho(r), r)_{s, n} & \leq \sum_{1 \leq|\alpha| \leq n}\left(-c\left\|D^{\alpha} r\right\|_{s+\frac{d}{2}}^{2}+C\|r\|_{s+|\alpha|}^{2}+\left\|G_{\alpha}(r)\right\|_{s-\frac{d}{2}}\|r\|_{s+\frac{d}{2}+|\alpha|}\right) \\
& \leq-c\|r\|_{s+\frac{d}{2}+n}^{2}+C_{s, n}\|r\|_{s+n}^{2}+\varepsilon\|r\|_{s+\frac{d}{2}+n}^{2}+C\|r\|_{s+\frac{d}{2}+n} \\
& \leq C_{s, n}\left(\|r\|_{s+n}^{2}+1\right)
\end{aligned}
$$

if $\varepsilon$ is chosen sufficiently small.

To show assertion (ii), we use (32) and estimate (23). This yields

$$
(\rho(r), r)_{s, n} \leq \sum_{1 \leq|\alpha| \leq n}\left(C_{s}\|r\|_{s+|\alpha|}^{2}+\left\|G_{\alpha}(r)\right\|_{s}\|r\|_{s+|\alpha|}\right) \leq C_{s, n}\left(\|r\|_{s+n}^{3}+1\right) .
$$

Thus the lemma is proved

Based on these a priori estimates, we are able to show an existence result for the solution of problem (21). For $T>0$ and $X$ a Banach space, we will denote by $C_{w}([0, T], X)$ and $C_{w}^{1}([0, T], X)$ the spaces of weakly continuous and weakly differentiable functions $u:[0, T] \longrightarrow X$, i.e. the functions for which $\langle\varphi, u\rangle \in C([0, T])$ and $\langle\varphi, u\rangle \in C^{1}([0, T])$ for all $\varphi \in X^{\prime}$, respectively.

Theorem 1 (Short-time existence of solutions). Let the assumptions of Lemma 7 (i) or (ii) be fulfilled. There are constants $\varepsilon>0$ and $T>0$ such that for all $n \geq d$ and all

$$
r_{0} \in B_{0}\left(\varepsilon, H^{s+d}\left(S^{N-1}\right) \cap H^{s+n}\left(S^{N-1}\right)\right)
$$

there is a solution

$$
r \in C_{w}\left([0, T], H^{s+n}\left(S^{N-1}\right)\right) \cap C_{w}^{1}\left([0, T], H^{s+n-d}\left(S^{N-1}\right)\right)
$$

of problem (21) satisfying $r(t) \rightarrow r_{0}$ in $H^{s+n}\left(S^{N-1}\right)$ as $t \rightarrow 0$.

There is at most one solution of problem (21) in

$$
C^{1}\left([0, T], H^{s+d}\left(S^{N-1}\right)\right) \cap L^{\infty}\left((0, T), H^{s+\frac{3}{2} d}\left(S^{N-1}\right)\right) .
$$

The existence result can be proved by a Galerkin approximation as in 121: Proof of Theorem A]. For the details of this as well as for the uniqueness result we refer to [18: Proofs of Propositions 1 and 2] or [26: Proof of Theorem 1] where the same abstract result is proved in the context of special applications. We emphasize that the choice of $\varepsilon$ and $T$ is independent of $n$, i.e. the smoothness of the initial datum is preserved during the evolution, even if the initial datum is small only in a weaker norm.

For parabolic equations, however, one expects a smoothing effect of the evolution. More precisely, the following result will be shown by a bootstrapping argument. 
Theorem 2 (Smoothing property for parabolic equations). Suppose

$$
-\rho^{\prime}(0) \in \mathcal{H}\left(H^{s+d+1}\left(S^{N-1}\right), H^{s+1}\left(S^{N-1}\right)\right) \cap \mathcal{H}\left(H^{s+d}\left(S^{N-1}\right), H^{s}\left(S^{N-1}\right)\right) .
$$

Then there are numbers $\varepsilon>0$ and $T>0$ such that for any solution

$$
r \in C^{1}\left([0, T], H^{s+d+1}\left(S^{N-1}\right)\right)
$$

of problem (21) that satisfies $r(0) \in B_{0}\left(\varepsilon, H^{s+d+1}\left(S^{N-1}\right)\right)$ we have

$$
r \in C^{\infty}\left((0, T], C^{\infty}\left(S^{N-1}\right)\right)
$$

Proof. It is sufficient to show that

$$
r \in C^{1}\left((0, T], C^{\infty}\left(S^{N-1}\right)\right)
$$

Indeed, this implies $r \in C^{1}\left((0, T], H^{9+2 k d}\left(S^{N-1}\right)\right)$ for any $k \in \mathbb{N}$, and using the fact that $\rho$ maps $\mathcal{W} \cap H^{s+j d}\left(S^{N-1}\right)$ smoothly into $H^{s+(j-1) d}\left(S^{N-1}\right)$ for $j \geq 1$ we get from $\dot{r}(t)=\rho(r(t))$ that $r \in C^{k+1}\left((0, T], H^{s+k d}\left(S^{N-1}\right)\right)$. As $k$ is arbitrary, this implies the assertion.

If $\varepsilon$ and $T$ are chosen small enough, then $\rho^{\prime}(r(t))-\rho^{\prime}(0)$ is small in

$$
\mathcal{L}\left(H^{s+d+1}\left(S^{N-1}\right), H^{s+1}\left(S^{N-1}\right)\right) \cap \mathcal{L}\left(H^{s+d}\left(S^{N-1}\right), H^{s}\left(S^{N-1}\right)\right)
$$

hence, by a well-known perturbation result,

$$
\begin{aligned}
-A(t) & =-\rho^{\prime}(r(t)) \\
& \in \mathcal{H}\left(H^{s+d+1}\left(S^{N-1}\right), H^{s+1}\left(S^{N-1}\right)\right) \cap \mathcal{H}\left(H^{s+d}\left(S^{N-1}\right), H^{s}\left(S^{N-1}\right)\right)
\end{aligned}
$$

for all $t \in[0, T]$. We choose $\delta \in(0, T), \gamma \in(0,1)$ and a strictly increasing sequence $\left\{\delta_{k}\right\} \subset(0, \delta)$ arbitrarily. To show (33) we prove

$$
D^{\beta} r \in C^{1}\left(\left[\delta_{k}, T\right], H^{s+1}\left(S^{N-1}\right)\right) \cap C^{\gamma}\left(\left[\delta_{k}, T\right], H^{s+d+1}\left(S^{N-1}\right)\right) \quad(|\beta| \leq k)
$$

by induction over $k \in \mathbb{N}$. For $k=0$, (34) holds by assumption. Suppose now (34) holds for $k=n$. This implies

$$
r \in C^{1}\left(\left[\delta_{n}, T\right], H^{s+n+1}\left(S^{N-1}\right)\right) \cap C^{\gamma}\left(\left[\delta_{n}, T\right], H^{s+n+d+1}\left(S^{N-1}\right)\right)
$$

We pick an arbitrary $\alpha$ with $|\alpha|=n+1$ and set $u=D^{\alpha} r$. By Lemma 3 we get that $u \in C^{1}\left(\left[\delta_{n}, T\right], H^{s}\left(S^{N-1}\right)\right)$ satisfies

$$
\left.\begin{array}{rl}
\dot{u} & =A(t) u+f(t) \\
u\left(\delta_{n}\right) & =D^{\alpha} r\left(\delta_{n}\right)
\end{array}\right\}
$$


where, by our assumptions,

$$
\begin{aligned}
A(t)=\rho^{\prime}(r(t)) \in & C^{\gamma}\left(\left[\delta_{n}, T\right], \mathcal{L}\left(H^{s+d}\left(S^{N-1}\right), H^{s}\left(S^{N-1}\right)\right)\right) \\
& \cap C^{\gamma}\left(\left[\delta_{n}, T\right], \mathcal{L}\left(H^{s+d+1}\left(S^{N-1}\right), H^{s+1}\left(S^{N-1}\right)\right)\right) \\
f(t)= & G_{\alpha}(r(t)) \in C^{\gamma}\left(\left[\delta_{n}, T\right], H^{s+1}\left(S^{N-1}\right)\right) .
\end{aligned}
$$

A standard result on non-autonomous linear parabolic equations (see, e.g., [23: Theorem 6.1.4] for a stronger result) yields

$$
u \in C^{1}\left(\left[\frac{\delta_{n}+\delta_{n+1}}{2}, T\right], H^{s}\left(S^{N-1}\right)\right) \cap C^{\gamma}\left(\left[\frac{\delta_{n}+\delta_{n+1}}{2}, T\right], H^{s+d}\left(S^{N-1}\right)\right) .
$$

In particular, as $d \geq 1$ we have $u\left(\frac{\delta_{n}+\delta_{n+1}}{2}\right) \in H^{s+1}\left(S^{N-1}\right)$. Applying the same arguments to the initial value problem

$$
\left.\begin{array}{rl}
\dot{u} & =A(t) u+f(t) \\
u\left(\frac{\delta_{n}+\delta_{n+1}}{2}\right) & =D^{\alpha} r\left(\frac{\delta_{n}+\delta_{n+1}}{2}\right)
\end{array}\right\}
$$

yields

$$
D^{\alpha} r \in C^{1}\left(\left[\delta_{n+1}, T\right], H^{s+1}\left(S^{N-1}\right)\right) \cap C^{\gamma}\left(\left[\delta_{n+1}, T\right], H^{s+d+1}\left(S^{N-1}\right)\right)
$$

which completes the induction proof

\section{Applications}

The abstract framework given in the previous sections reduces the analysis of problem (21) (as far as short-time existence is concerned) to the verification of (17) and the study of the lincarization $\bar{\rho}^{\prime}(R)$. In the case of parabolic problems, the aim is to show the validity of the assumptions of Lemma 5 for $s>s_{0}$. Then Lemmas 6 and $7 /(\mathrm{i})$ are applicable, and thus Theorems 1 and 2 hold.

Our first applications are extensions of the results obtained in $[18,26]$.

7.1 Stokes flow driven by surface tension. We return to the example considered in Section 3. Lemma 2 shows that in the situation described there, (17) is satisfied with $K=\omega$ and $d=1$. It remains to consider the linearization $\bar{\rho}(0)$. Straightforward calculations that have been carried out in [18] give

$$
\bar{\rho}^{\prime}(0)[h]=n^{T} B n \Delta_{\Gamma_{0}} h+L_{1} h+L_{0} h,
$$

where $L_{0} \in \mathcal{L}\left(H^{s}\left(\Gamma_{0}\right)\right)$ for $s>s_{0}=\frac{N+3}{2}, L_{1}$ is a first-order differential operator corresponding to a smooth vector field, and

$$
B \in \mathcal{L}\left(\left(H^{\frac{1}{2}}\left(\Gamma_{0}\right)\right)^{N},\left(H^{\frac{3}{2}}\left(\Gamma_{0}\right)\right)^{N}\right)
$$


is given as $B \varphi=\left.u\right|_{\Gamma_{0}}$ where $(u, p, \lambda)$ is the (uniquely determined) solution of the StokesNeumann problem

$$
\left.\begin{array}{rlrl}
-\Delta u+\nabla p+\lambda_{1} & =0 & & \text { in } \Omega_{0} \\
\operatorname{div} u & =0 & & \text { in } \Omega_{0} \\
\tau(u, p) n+\lambda_{2} \otimes n & =\varphi & & \text { on } \Gamma_{0} \\
\int_{\Omega_{0}} u d x & =0 & & \\
\int_{\Omega_{0}} \operatorname{rot} u d x & =0 . &
\end{array}\right\}
$$

It follows from the general theory of elliptic systems that

$$
A=n^{T} B n \Delta_{\Gamma_{0}}+L_{1}
$$

is a pseudodifferential operator of order 1 for which the coercivity estimate (29) can be shown either on the basis of the weak formulation of (36), using Korns second inequality (see [18: Section 5]) or by explicit calculation of the principal symbol and application of Gårdings inequality.

Summarizing our main results and using a compactness argument again, we obtain the following theorem.

Theorem 3 (Well-posedness and smoothness of the boundary for Stokes flow driven by surface tension). Let $\mathcal{U}(\Omega)=\left.u\right|_{a \Omega}$ where u solves problem (8), $\tilde{s}>s>$ $s_{0}=\frac{N}{2}+2$. There are constants $\varepsilon>0$ and $T>0$ depending only on $\Phi, \tilde{s}$, and $s$ such that for any

$$
r_{0} \in B_{0}\left(\varepsilon, H^{s+2}\left(S^{N-1}\right)\right) \cap H^{j+3}\left(S^{N-1}\right)
$$

there is a unique solution

$$
r \in C\left([0, T], H^{s+3}\left(S^{N-1}\right)\right) \cap C^{1}\left([0, T], H^{s+2}\left(S^{N-1}\right)\right)
$$

of the initial value problem (21). Moreover,

$$
r \in C^{\infty}\left((0, T), C^{\infty}\left(S^{N-1}\right)\right)
$$

and if $r_{0} \in H^{j+n}\left(S^{N-1}\right)$ for an $n \in \mathbb{N}$, then $r \in C\left([0, T], H^{s+n}\left(S^{N-1}\right)\right)$.

Remark. Here as well as in the following applications, the smoothness of the initial perturbation $r_{0}$ is chosen high enough to obtain existence, uniqueness, and smoothness of the solution at once. This is not an optimal choice if one is interested in existence of solutions only.

7.2 Hele-Shaw flow driven by surface tension. The following moving boundary problem can be discussed in a completely analogous manner: Let $\mathcal{U}(\Omega)=\left.\nabla u\right|_{\text {an }}$ where $u$ is the solution of the standard elliptic boundary value problem

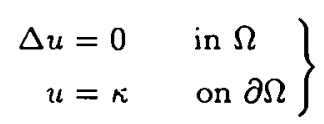


where $\kappa$ is defined as in Section 3. This is a formulation for the so-called (one-phase) Hele-Shaw flow problem with surface tension. In the case $N=2$, it occurs as a model for the motion of a blob of liquid confined in the thin gap between two plane parallel plates. For $N=2$ and $N=3$ it also describes the motion of phase boundaries by capillarity and volume diffusion in metallurgy [25]. For $N=2$, global solvability in time has been shown in [10] for initial data near the equilibria which are given by the balls. Moreover, exponential stability of these equilibria is proved. Short-time solvability results for the moving boundary problem in the case where $\Omega(t)$ is a perturbed halfspace in $\mathbb{R}^{2}$ are established in [12] and, in a more general situation including also the influence of sources and sinks, in [22]. A corresponding two-phase problem is discussed in [9]. Furthermore, the linearization of the one-phase problem is briefly discussed in [2].

Our analysis is in many aspects similar to the onc given in [15], where the theory of maximal regularity in little Hölder spaces is used to show existence, uniqueness, and $C^{\infty}$-smoothness of solutions for a related problem where $\partial \Omega(t)$ consists of a fixed and a moving component. Due to the analytic setting, some parts of the proofs are considerably more involved than the ones given here. As pointed out above, we generalize the results of [26].

Using the well-known regularity results for (37) and the same perturbation arguments as in the proof of Lemma 2, one shows that (17) holds with $d=3, K=\omega$, and $s>s_{0}=\frac{N+1}{2}$. The linearization has been carried out in [26], it yields

$$
\bar{\rho}^{\prime}(0)=A_{D N} \Delta_{\Gamma_{0}}+L_{2},
$$

where $L_{2} \in \mathcal{L}\left(H^{s+2}\left(\Gamma_{0}\right), H^{s}\left(\Gamma_{0}\right)\right)$ and $A_{D N}$ is the so-called Dirichlet-Neumann operator on $\Omega_{0}$ which maps $\varphi$ to the outer normal derivative $A_{D N} \varphi=\frac{\partial u}{\partial n}$ where $u$ solves

$$
\left.\begin{array}{rl}
\Delta u=0 & \text { in } \Omega_{0} \\
u=\varphi & \text { on } \partial \Omega_{0} .
\end{array}\right\}
$$

Again, the general theory of elliptic equations yields that $A=A_{D N} \Delta_{\Gamma_{0}}$ is a pseudodifferential operator of order $d=3$, and the coercivity estimate (29) is shown in [26: Lemma 8]. (Alternatively, it can be obtained by calculation of the principal symbol and Gårdings inequality also in this situation.)

Summarizing, we find the following result.

Theorem 4 (Well-posedness and smoothness of the boundary for Hele-Shaw flow driven by surface tension). Let $\mathcal{U}(\Omega)=\left.\nabla u\right|_{\text {an }}$ where u solves problem $(37), \tilde{s}>s>$ $s_{0}=\frac{N}{2}+2$. There are constants $\varepsilon>0$ and $T>0$ depending only on $\Phi, \tilde{s}$, and $s$ such that for any

$$
r_{0} \in B_{0}\left(\varepsilon, H^{s+4}\left(S^{N-1}\right)\right) \cap^{\prime} H^{\bar{s}+7}\left(S^{N-1}\right)
$$

there is a unique solution

$$
r \in C\left([0, T], H^{s+7}\left(S^{N-1}\right)\right) \cap C^{1}\left([0, T], H^{s+4}\left(S^{N-1}\right)\right)
$$

of the initial value problem (21). Moreover,

$$
r \in C^{\infty}\left((0, T), C^{\infty}\left(S^{N-1}\right)\right)
$$


and if $r_{0} \in H^{j+n}\left(S^{N-1}\right)$ for an $n \in \mathbb{N}$, then $r \in C\left([0, T], H^{s+n}\left(S^{N-1}\right)\right)$.

7.3 Classical Hele-Shaw flow. To describe the classical Hele-Shaw flow problem, we consider the elliptic boundary value problem

$$
\left.\begin{array}{rlr}
-\Delta v=f & \text { in } \Omega \\
v=0 & & \text { on } \partial \Omega
\end{array}\right\}
$$

In order to include various interior driving mechanisms, as distributed sources and sinks, point sources and sinks, as well as multipoles of arbitrary order, we allow a very general right-hand side, namely, $f \in \mathcal{E}^{\prime}\left(\Omega_{0}\right)$, i.e. $f$ is a distribution with compact support in $\Omega_{0}$. This implies that in general $v \in \mathcal{D}^{\prime}\left(\Omega_{0}\right)$. Outside supp $f$, however, $v$ is represented by an analytic function, and thus the Dirichlet boundary condition makes sense if $\partial \Omega$ is near $\partial \Omega_{0}$. As before, the motion of the domain is given by $\mathcal{U}(\Omega)=\left.\nabla v\right|_{\partial \Omega}$. The condition for well-posedness which has to be imposed on $f$ is

$$
\frac{\partial v_{0}}{\partial n}>0 \quad \text { on } \partial \Omega_{0}
$$

for the solution $v_{0}$ of problem (38) with $\Omega=\Omega_{0}$. This means that we consider a situation in which the liquid domain is everywhere advancing. By the strong maximum principle, (39) is automatically satisfied if $f$ is a positive measure, as in $[6,20]$.

We set $g=G * f$ where $G$ denotes a fundamental solution of the Laplacian. The function $g$ is analytic outside supp $f$. Setting $u=v-g$ we have $\mathcal{U}(\Omega)=\left.\nabla(u+g)\right|_{a \Omega}$ and

$$
\left.\begin{array}{rlrl}
\Delta u & =0 & & \text { in } \Omega \\
u & =-g & & \text { on } \partial \Omega .
\end{array}\right\}
$$

Transforming (40) from $\Omega=\Omega_{\theta, r}$ to $B_{0}$ yields

$$
L(\theta, r) u(\theta, r)=\left[\begin{array}{c}
0 \\
\left.\left(-g \circ \Phi_{\theta, r}\right)\right|_{S^{N-1}}
\end{array}\right]
$$

where

$$
u(\theta, r)=u \circ \Phi_{\theta, r}=\Phi_{\theta, r}^{*} u \quad \text { and } \quad L(\theta, r)=\left[\begin{array}{c}
\Phi_{\theta, r}^{*} \Delta\left(\Phi_{\theta, r}^{*}\right)^{-1} \\
\operatorname{Tr}_{S^{N-1}}
\end{array}\right]
$$

The mapping $\left.(\theta, r) \mapsto\left(-g \circ \Phi_{\theta, r}\right)\right|_{S^{N-1}}$ is analytic from a small neighborhood of 0 in

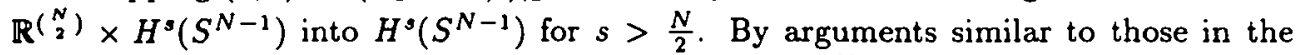
proof of Lemma 2, we conclude that (17) holds for $s>\frac{N+1}{2}$ and $d=1$.

To calculate $\bar{\rho}^{\prime}(0)$ we perform a change of coordinates as in Section 5 and find

$$
\bar{\rho}(R)=\frac{\left.B(R)\left(\bar{u}(R)+\dot{\bar{\psi}}(R)^{*} g\right)\right) \mid \Gamma_{0} \cdot \bar{\nu}(R)}{\bar{n} \cdot \bar{\nu}(R)}
$$


where

$$
\begin{aligned}
\bar{\psi}(R) & =\Phi_{0, r(R)} \circ \Phi^{-1} \\
\bar{\psi}(R)^{*} g & =g \circ \bar{\psi}(R) \\
\bar{\nu}(R) & =n_{\Omega_{0, r(R)} \circ \bar{\psi}(R)} \\
\bar{u}(R) & =u(0, r(R)) \circ \Phi^{-1} \\
\bar{n} & =\bar{\nu}(0) \\
B(R) v & =\left(\nabla\left(v \circ \bar{\psi}(R)^{-1}\right)\right) \circ \bar{\psi}(R),
\end{aligned}
$$

the mapping $R \mapsto r(R)$ denotes the inverse of the mapping $r \mapsto R(r)$ defined by (25), and $n_{\Omega}$ denotes the outer unit normal field on $\partial \Omega$. Note the $\bar{\psi}(0)=\mathrm{id}_{\Omega_{0}}$ and hence

$$
\left((\partial \bar{\psi})^{-1}\right)^{\prime}(0)=-(\partial \bar{\psi})^{\prime}(0)
$$

where $\partial \bar{\psi}$ denotes the Jacobian of $\bar{\psi}$. Consequently, we find by straightforward calculations

$$
B^{\prime}(0)[h]=(\vec{\psi}(0)[h] \cdot \nabla) \circ \nabla-\nabla \circ\left(\bar{\psi}^{\prime}(0)[h] \cdot \nabla\right)
$$

and, using that $\left.\vec{\psi}(0)[h]\right|_{\Gamma_{0}}=h \bar{n}$,

$$
\begin{aligned}
\bar{\rho}^{\prime}(0)[h]= & \frac{\partial^{2}}{\partial n^{2}}(\bar{u}(0)+g) h-\frac{\partial}{\partial n}\left(\bar{\psi}^{\prime}(0)[h] \cdot \nabla \bar{u}(0)\right)+\frac{\partial}{\partial n}\left(\bar{u}^{\prime}(0)[h]\right) \\
& +\left.(\nabla(\bar{u}(0)+g))\right|_{\Gamma_{0}} \cdot \nu^{\prime}(0)[h] .
\end{aligned}
$$

It remains to calculate $\bar{u}^{\prime}(0)[h]$ from

$$
\bar{L}(R) \bar{u}(R)=\left[\begin{array}{c}
0 \\
-\left.\bar{\psi}(R)^{*} g\right|_{\Gamma_{0}}
\end{array}\right]
$$

where

$$
\bar{L}(R)=\left[\begin{array}{c}
P(R) \\
\operatorname{Tr}_{\Gamma_{0}}
\end{array}\right] \quad \text { and } \quad P(R) v=\left(\Delta\left(v \circ \bar{\psi}(R)^{-1}\right)\right) \circ \bar{\psi}(R) .
$$

By similar calculations as for (41) we find

$$
P^{\prime}(0)[h]=\left(\bar{\psi}^{\prime}(0)[h] \cdot \nabla\right) \circ \Delta-\Delta \circ(\vec{\psi}(0)[h] \cdot \nabla)
$$

and thus

$$
\begin{aligned}
\bar{u}^{\prime}(0)[h] & =-\bar{L}(0)^{-1} \bar{L}_{1}(0)\left[h \mid \bar{L}(0)^{-1}\left[\begin{array}{c}
0 \\
g \mid \Gamma_{0}
\end{array}\right]+\bar{L}(0)^{-1}\left[\begin{array}{c}
0 \\
-\frac{\partial g}{\partial n} h
\end{array}\right]\right. \\
& =\bar{L}(0)^{-1}\left[\begin{array}{c}
\Delta\left(\bar{\psi}^{\prime}(0)[h] \cdot \nabla \bar{u}(0)\right) \\
-\frac{\partial g}{\partial n} h
\end{array}\right]
\end{aligned}
$$

because $\bar{L}(0)^{-1}\left[\begin{array}{c}0 \\ g \mid r_{0}\end{array}\right]=\bar{u}(0)$ and the first term in $P^{\prime}(0)[h] \bar{u}(0)$ vanishes. Therefore

$$
\bar{u}^{\prime}(0)[h]=\bar{\psi}^{\prime}(0)[h] \cdot \nabla \bar{u}(0)+w[h]
$$


where $w=w[h]$ solves

$$
\left.\begin{array}{rrr}
\Delta w & =0 & \text { in } \Omega_{0} \\
w=\left(-\frac{\partial g}{\partial n}-\frac{\partial \bar{u}(0)}{\partial n}\right) h & \text { on } \Gamma_{0} .
\end{array}\right\}
$$

Thus we obtain, writing $v_{0}=\bar{u}(0)+\left.g\right|_{\Omega_{0}}$,

$$
\bar{\rho}^{\prime}(0)[h]=A_{D N}\left(-\frac{\partial v_{0}}{\partial n} h\right)+\frac{\partial^{2} v_{0}}{\partial n^{2}} h+\nabla v_{0} \cdot \bar{\nu}^{\prime}(0)[h]
$$

with $A_{D N}$ as defined in the previous subsection. $A_{D N}$ is known to be a pseudodifferential operator of order 1 on the manifold $\Gamma_{0}$, and therefore the commutator properties of operators of this kind yield

$$
\bar{\rho}^{\prime}(0)=-\frac{\partial v_{0}}{\partial n} A_{D N}+D+K
$$

where $K$ is a pseudodifferential operator of order 0 on $\Gamma_{0}$ and $D=\nabla v_{0} \cdot \bar{\nu}^{\prime}(0)$ is a firstorder differential operator corresponding to a smooth vector field on $\Gamma_{0}$. This implies that the operator $D+D^{*}$ is given just by multiplication with a smooth function, and consequently we have

$$
((D+K) u, u)_{0}^{\Gamma_{0}}=\left(\left(\frac{1}{2}\left(D+D^{*}\right)+K\right) u, u\right)_{0}^{\Gamma_{0}} \leq C\|u\|_{0}^{2} \quad\left(u \in H^{1}\left(\Gamma_{0}\right)\right) .
$$

Elementary arguments based on the Green formula and the Poincaré inequality show that

$$
\left(A_{D N} u, u\right)_{0}^{\Gamma_{0}} \geq c\|u\|_{\frac{1}{2}}^{2}-C\|u\|_{-\frac{1}{2}}^{2} \quad\left(u \in H^{1}\left(\Gamma_{0}\right)\right)
$$

with a constant $c>0$ depending on $\Gamma_{0}$ only (see [26: Equation (4.2)]). Combining (39) and (43) - (45) we find

$$
\left(\bar{\rho}^{\prime}(0)[h], h\right)_{0}^{\Gamma_{0}} \leq-c\|h\|_{\frac{1}{2}}^{2}+C\|h\|_{-\frac{1}{2}}^{2} \quad\left(h \in H^{1}\left(\Gamma_{0}\right)\right)
$$

and by the commutator property $\vec{\rho}^{\prime}(0) \Lambda_{\Gamma_{0}}^{s}-\Lambda_{\Gamma_{0}}^{s} \bar{\rho}^{\prime}(0) \in \mathcal{L}\left(H^{s}\left(\Gamma_{0}\right), H^{0}\left(\Gamma_{0}\right)\right)$

$$
\left(\bar{\rho}^{\prime}(0)[h], h\right)_{s}^{\Gamma_{0}} \leq-c\|h\|_{s+\frac{1}{2}}^{2}+C\|h\|_{s}^{2} \quad\left(h \in H^{s+1}\left(\Gamma_{0}\right)\right) .
$$

Hence the assumptions of Lemma 5 are satisfied with $d=1$ and $A=-\frac{\partial v_{0}}{\partial n} A_{D N}+D$.

Summarizing, we find the following result.

Theorem 5 (Well-posedness and smoothness of the boundary for Hele-Shaw flow with advancing liquid front). Let $\mathcal{U}(\Omega)=\left.\nabla v\right|_{\text {an }}$ where $v$ solves problem $(38), \tilde{s}>s>$ $s_{0}=\frac{N}{2}+1$. Suppose that (39) holds. Then there are constants $\varepsilon>0$ and $T>0$ depending only on $\Phi, f, \tilde{s}$, and s such that for any

$$
r_{0} \in B_{0}\left(\varepsilon, H^{s+2}\left(S^{N-1}\right)\right) \cap H^{3+3}\left(S^{N-1}\right)
$$


there is a unique solution

$$
r \in C\left([0, T], H^{s+3}\left(S^{N-1}\right)\right) \cap C^{1}\left([0, T], H^{s+2}\left(S^{N-1}\right)\right)
$$

of the initial value problem (21). Moreover,

$$
r \in C^{\infty}\left((0, T), C^{\infty}\left(S^{N-1}\right)\right)
$$

and if $r_{0} \in H^{\bar{s}+n}\left(S^{N-1}\right)$ for an $n \in \mathbb{N}$, then $r \in C\left([0, T], H^{s+n}\left(S^{N-1}\right)\right)$.

Remarks. The results of this subsection can be seen as a gencralization of the results in $[6,20]$ with respect to the driving mechanisms. Our result are weaker in the sense that we do not prove analyticity of $\partial \Omega(t)$ for $t>0$. Note, however, that (in contrast to the earlier results mentioned above) we do obtain smoothness of the evolution of the interface in time.

The linearization (42) corresponds to the results which are obtained in [2: Lemma 2.1] in a somewhat informal manner and a slightly different analytic setting. Furthermore, the condition (4.i) for (linear) well-posedness of a Hele-Shaw type problem given in [2] corresponds to our condition (39). This condition is sharp in the sense that the linearized operator loses its coercivity if (39) is violated. For corresponding results in a different geometry, see [17]. Note that, by the strong maximum principle, the condition on the function $b$ in Theorem 1.1 there implies the validity of a condition like (39) on the moving component of the boundary.

Acknowledgments. The author is indebted to Prof. J. Escher and Prof. M. Günther for a lot of fruitful discussions.

\section{References}

[1] Abergel, F.: A geometric approach to the study of stationary free surface flows for viscous liquids. Proc. Royal Soc. Edinburgh 123A (1993), 209 - 229.

[2] A bergel, F.: Well-posedness for a Cauchy problem associated to time-dependent free boundaries with nonlocal leading terms. Comm. Part. Diff. Equ. 21 (1996), $1307-1319$.

[3] Angenent, S.: Nonlinear analytic semiflows. Proc. Roy. Soc. Edinburgh 115A (1990), 91 - 107.

[4] Angenent, S.: Parabolic equations for curves on surfaces. Part I: Curves with p-integrable curvature. Ann. Math. 132 (1990), $451-483$.

[5] Antanovskii, L.K.: Creeping thermocapillary motion of a two-dimensional deformable bubble: existence theorem and numerical simulation. Eur. J. Mech., B/Fluids 11 (1992), 741 $-758$.

[6] Begehr, H. and R. P. Gilbert: Hele-Shaw type flows in $\mathbb{R}^{n}$. Nonlin. Analysis: TMA 10 (1986), $65-85$.

[7] Bemelmans, J.: Gleichgewichtsfiguren zäher Flüssigkeiten mit Oberfï̈chenspannung. Anal. 1 (1981), $241-282$.

[8] Beyer, K. and M. Günther: On the Cauchy problem for a capillary drop. Part I: Irrotational motion (to appear). 
[9] Chen, X.: The Hele-Shaw problem and area-preserving curve-shortening motions. Arch. Rat. Mech. Anal. 123 (1993), $117-151$.

[10] Constantin, P. and M. Pugh: Global solution for small data to the Hele-Shaw problem. Nonlinearity 6 (1993), $393-415$.

[11] Dierkes, U., Hildebrandt, S., Küster, A. and O. Wohlrab: Minimal surfaces, Vol. I. Berlin: Springer 1992.

[12] Duchon, J. and R. Robert: Évolution d'une interface par capillarité et diffusion. De volume I: Existence locale en temps. Ann. Inst. Henri Poincaré Analyse non linéaire 1 (1984), $361-378$.

[13] Eidel'man, S.D.: Parabolic Systems. Amsterdam - London: North-Holland 1969.

[14] Elst, A.F.M. ter and D. W. Robinson: Subcoercivity and subelliptic operators on Lie groups. Part I: Free nilpotent groups. Potential analysis 3 (1994), 283 - 337.

[15] Escher, J. and G. Simonett: Classical solutions for Hele-Shaw models with surface tension. Advances Diff. Equ. 2 (1997), $619-642$.

[16] Escher, J. and G. Simonett: Analyticity of the interface in a free boundary problem. Math. Ann. 305 (1996), $439-459$.

[17] Escher, J. and G. Simonett: Classical solutions for multidimensional Hele-Shaw models. SIAM J. Math. Anal. 28 (1997), 1028 - 1047.

[18] Gūnther, M. and G. Prokert: Existence results for the quasistationary motion of a capillary liquid drop. Z. Anal. Anw. 16 (1997), $311-348$.

[19] Günther, M.: Private communication.

[20] Gustafsson, B.: Applications of variational inequalities to a moving boundary problem for Hele-Shaw flows. SIAM J. Math. Anal. 16 (1985), 279 - 300.

[21] Kato, T. and C. Y. Lai: Nonlinear evolution equations and the Euler flow. J. Funct. Anal. 56 (1984), $15-28$.

[22] Kimura, M.: Time-local existence of a moving boundary of the Hele-Shaw flow with suction. Report. Hiroshima: Univ. 1998, INSAM Report No. 36 (98-03).

[23] Lunardi, A.: Analytic Semigroups and Optimal Regularity in Parabolic Problems. Basel: Birkhäuser 1995.

[24] Martin, R.H.: Nonlinear Operators and Differential Equations in Banach Spaces. New York: Wiley-Intersci. 1976.

[25] Mullins, W. W.: Grain boundary grooving by volume diffusion. Trans. Metallurgical Soc. AIME 218 (1960), 354 - 361.

[26] Prokert, G.: Existence results for Hele-Shaw flow driven by surface tension. European J. Appl. Math. 9 (1998), $195-221$.

[27] Zeidler, E.: Nonlinear Functional Analysis and Its Applications. New York: Springer 1988. 